\title{
BOUNDARY ELEMENT MONOTONE ITERATION SCHEME FOR SEMILINEAR ELLIPTIC PARTIAL DIFFERENTIAL EQUATIONS
}

\author{
YUANHUA DENG, GOONG CHEN, WEI-MING NI, AND JIANXIN ZHOU
}

\begin{abstract}
The monotone iteration scheme is a constructive method for solving a wide class of semilinear elliptic boundary value problems. With the availability of a supersolution and a subsolution, the iterates converge monotonically to one or two solutions of the nonlinear PDE. However, the rates of such monotone convergence cannot be determined in general. In addition, when the monotone iteration scheme is implemented numerically through the boundary element method, error estimates have not been analyzed in earlier studies. In this paper, we formulate a working assumption to obtain an exponentially fast rate of convergence. This allows a margin $\delta$ for the numerical implementation of boundary elements within the range of monotone convergence. We then interrelate several approximate solutions, and use the Aubin-Nitsche lemma and the triangle inequalities to derive error estimates for the Galerkin boundaryelement iterates with respect to the $H^{r}(\Omega), 0 \leq r \leq 2$, Sobolev space norms. Such estimates are of optimal order. Furthermore, as a peculiarity, we show that for the nonlinearities that are of separable type, "higher than optimal order" error estimates can be obtained with respect to the mesh parameter $h$. Several examples of semilinear elliptic partial differential equations featuring different situations of existence/nonexistence, uniqueness/multiplicity and stability are discussed, computed, and the graphics of their numerical solutions are illustrated.
\end{abstract}

\section{INTRODUCTION}

Numerical solutions of nonlinear partial differential equations (PDEs) are important in applications. Historically, such work is done primarily by the finite difference methods (FDM) and finite element methods (FEM). While boundary element methods (BEM) have steadily gained popularity among engineers and scientists in their work of computing solutions of PDEs, owing to the very nature of their formulation, BEM are still regarded by many people as mainly applicable to

Received by the editor May 4, 1994 and, in revised form, March 1, 1995.

1991 Mathematics Subject Classification. Primary 31B20, 35J65, 65N38.

Key words and phrases. Numerical PDE, boundary elements, potential theory, nonlinear PDE, elliptic type.

The first, second, and fourth authors were supported in part by AFOSR Grant 91-0097 and NSF Grant DMS 9404380.

The third author was supported in part by NSF Grants DMS 9101446 and 9401333.

The second author was on sabbatical leave at the Institute of Applied Mathematics, National Tsing Hua University, Hsinchu, Taiwan, ROC. Supported in part by a grant from the National Science Council of the Republic of China. 
linear PDEs. Nevertheless, progress has been made; several researchers have applied BEM to encompass nonlinearities in elliptic boundary value problems (BVPs) with some success. For example,

(i) when the governing equation itself is linear, with nonlinearities occurring only in the boundary conditions, then the applicability of BEM is quite ready. A nonlinear integral equation can be formulated on the boundary; an essential feature and an advantage of BEM are retained. Error analysis for such problems has been done by Ruotsalainen and Wendland [15], e.g.;

(ii) when the governing equation is nonlinear, with the nonlinearities occurring in the lowest-order terms (leading to a semilinear PDE), Brebbia and Walker [5], and Sakakihara [16] have considered iteration schemes for such problems. Accurate numerical results for certain examples have been obtained. But no error analysis and convergence for the discretized scheme were established in [5] and [16].

The semilinear elliptic PDEs considered in [5] and [16] are of the form

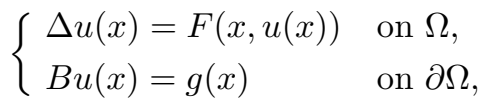

where $\Omega$ is a simply-connected bounded domain in $\mathbb{R}^{N}$ with $C^{\infty}$ smooth boundary $\partial \Omega, F: \bar{\Omega} \times \mathbb{R} \rightarrow \mathbb{R}$ is smooth such that $F(x, u)$ is nonlinear in $u, B$ is a linear boundary operator of the form

$$
B u=u \quad \text { or } \quad B u=\frac{\partial u}{\partial n}+\alpha(\cdot) u, \text { with } \alpha(x) \geq 0 \quad \forall x \in \partial \Omega,
$$

and $n$ is the unit outward normal on $\partial \Omega$. A straightforward iteration scheme suggested by Brebbia and Walker in [5] goes as follows:

$$
\left\{\begin{array}{l}
\text { Initialize: Take an initial guess } u_{0}(x) ; \\
\text { Iterate: Solve, for } n=0,1,2, \ldots \\
\begin{cases}\Delta u_{n+1}(x)=F\left(x, u_{n}(x)\right), & x \in \Omega, \\
\left(B u_{n+1}\right)(x)=g(x), & x \in \partial \Omega,\end{cases}
\end{array}\right.
$$

where at each iteration $u_{n+1}(\cdot)$ is solved by a boundary element method. Although this scheme appears quite natural and sometimes even produces nice numerical results, unfortunately, for "strong" nonlinearities, it fails to converge in general, as will be shown in $\S 2$.

On the other hand, the iteration scheme used by Sakakihara in [16] is the monotone iteration scheme:

(1) Choose a $\lambda>0$ "sufficiently large"; (cf. (2.9))

(2) Initialize: Take an initial guess $u_{0}(x)$;

(3) Iterate: Solve, for $n=0,1,2, \ldots$

$$
\left\{\begin{array}{l}
\Delta u_{n+1}(x)-\lambda u_{n+1}(x)=-\lambda u_{n}(x)+F\left(x, u_{n}(x)\right), \quad x \in \Omega, \\
\left(B u_{n+1}\right)(x)=g(x), \quad x \in \partial \Omega .
\end{array}\right.
$$

(Here $u_{n+1}$ is also solved by a boundary element method, but

(other general numerical methods may also be used.) 
The monotone iteration scheme is a well-known constructive method for solving semilinear elliptic PDEs of the type (1.1). When some supersolutions and subsolutions (also known as barriers) are available, monotone convergence of the iterates $u_{n+1}(x)$ (of the undiscretized scheme) can be established $[2,12,17]$. In [16], Sakakihara used the Green's formula, i.e., the commonly called direct approach, to formulate boundary integral equations (BIEs) to solve each $u_{n+1}(x)$. The convergence of boundary layer densities in those BIEs can be readily established, yielding the convergence of $u_{n}(x)$ to some $u(x)$ satisfying (1.1), i.e., a solution of (1.1). Nevertheless, in practical BEM computations, a BIE formulation of (1.4) must be discretized. Analysis of convergence and error for the discretized problem requires the knowledge of regularities of several singular integral operators involved in the BIEs. Such work is missing in [16].

In boundary element computations of PDEs, indirect formulations using only simple- or double-layer potentials to represent the solution are known to have several important advantages, one among which is a significant reduction of the amount of quadrature involved. Here, in our opinion, using the simple-layer potential representation (plus a volume potential to account for the right-hand side $-\lambda u_{n}(x)+F\left(x, u_{n}(x)\right)$ in (1.4), see the second integral in (3.8)) is preferable to the direct formulation adopted in [16]. This will be the basic approach taken in this paper. We will undertake the task of establishing convergence and error estimates for the discretized Galerkin boundary-element scheme corresponding to this approach. In our attempt to establish convergence, we are faced with some fundamental issues in the study of nonlinear PDEs: nonexistence of solutions, or the existence of multiple solutions, some of which are known to be unstable $[12,17]$. (Unstable solutions may be briefly explained as a saddle-point type critical point on the solution manifold, which must be obtained through a mountain-pass lemma [3] kind of argument and are thus unobtainable as solutions of a maximization/minimization variational problem. Or, unstable solutions may be regarded as an unstable equilibrium of a corresponding dynamic problem.) Only after these questions are properly understood can one talk about which solution the numerical iterates are converging to. Indeed, this may help explain why earlier workers had not been totally successful in deriving any error estimates or convergence for the boundary element monotone iteration scheme. Not surprisingly, a certain additional working assumption (see $[\mathrm{H}]$ in $\S 4$ ) is needed in order for the Galerkin boundary element scheme to converge. This assumption, although looking somewhat restrictive at first glance, is actually natural because of the needs to be able to estimate errors, especially after seeing several types of concrete examples of semilinear PDEs analyzed and computed in $\S 7$.

The organization of the paper is as follows:

(i) In $\S 2$, we provide some further background material and discussion.

(ii) In $\S 3$, we prove the antimonotone convergence property of simple-layer densities of the (undiscretized) boundary integral equations according to our formulation.

(iii) In $\S 4$, we establish convergence and error estimates for a Galerkin boundary element scheme, subject to the Dirichlet boundary condition. By comparing various intermediate approximate solutions, error estimates $\left\|u_{h}^{n}-u_{\infty}\right\|_{H^{r}(\Omega)}$ are obtained which are sharp for the $H^{r}(\Omega)$ Sobolev space norms, $0 \leq r \leq 2$.

(iv) A distinctive feature of BEM for semilinear PDEs is that we are able to obtain 
"higher than optimal order" error estimates with respect to $h$, provided that the nonlinearity is separable (cf. (5.1)). This is presented in $\S 5$.

(v) Error estimates for the semilinear PDE subject to the Neumann or Robin boundary conditions are discussed in $\S 6$.

(vi) In $\S 7$, some typical cases of semilinear elliptic PDEs are discussed and computed, with numerical solutions illustrated.

The collocation scheme is computationally more convenient than the Galerkin one. Our numerical results in $\S 7$ are obtained via the former instead of the latter. At this moment we are still trying to analyze errors for the collocation scheme. This, along with several other related problems, has been partially studied [8] and will appear elsewhere.

\section{A StRAightForWARD iteration SCHEME AND THE MONOTONE ITERATION SCHEME}

We first review the straightforward iteration scheme (1.3). We show through an ODE example that (1.3) does not converge in general.

Example 2.1. Consider

$$
\left\{\begin{array}{l}
\frac{d^{2}}{d x^{2}} u(x)-u^{3}(x)=0, \quad 0<x<1, \\
u(0)=u(1)=0 .
\end{array}\right.
$$

The differential operator $\frac{d^{2}}{d x^{2}}$ represents the simplest elliptic operator in one dimension. Multiplying the equation in (2.1) by $u$ and integrating by parts, we obtain

$$
\int_{0}^{1}\left[\left|u^{\prime}(x)\right|^{2}+u^{4}(x)\right] d x=0 .
$$

Therefore, (2.1) admits a unique solution that is trivial. (This trivial solution is, of course, stable.)

Let us find iterative solutions of (2.1) by (1.3):

$$
\left\{\begin{array}{l}
\text { Set } u_{0}(x)=C>0 ; \\
\text { Solve, for } n=0,1,2, \ldots \\
\left\{\begin{array}{l}
u_{n+1}^{\prime \prime}(x)=u_{n}^{3}(x), \\
u_{n+1}(0)=u_{n+1}(1)=0 .
\end{array} \quad x \in[0,1],\right.
\end{array}\right.
$$

For $n=0$, for example, we get

$$
\begin{aligned}
& u_{1}^{\prime \prime}(x)=C^{3} \\
& u_{1}(x)=\frac{C^{3}}{2} x^{2}+\alpha_{1} x+\beta_{1}, \\
& u_{1}(0)=u_{1}(1)=0 \Rightarrow \beta_{1}=0, \alpha_{1}=-\frac{C^{3}}{2},
\end{aligned}
$$

hence

$$
u_{1}(x)=\frac{C^{3}}{2}\left(x^{2}-x\right)
$$


In general, we have

$$
u_{n}(x)=\alpha_{n} x^{m_{n}}+\cdots,
$$

where the leading coefficient $\alpha_{n}$ and the power $m_{n}$ can be uniquely determined recursively:

$$
\left\{\begin{array}{l}
\alpha_{0}=C, \quad m_{0}=0, \\
\alpha_{n+1}=\frac{\alpha_{n}^{3}}{\left(3 m_{n}+2\right)\left(3 m_{n}+1\right)}, \quad m_{n+1}=3 m_{n}+2, \quad n=0,1,2, \ldots .
\end{array}\right.
$$

From (2.5), it is not difficult to show that if we choose $C>\frac{2}{3} k_{0}$ for any $k_{0} \geq 6$, then

$$
\alpha_{n}>k_{0}\left(m_{n}+\frac{2}{3}\right) \rightarrow \infty \quad \text { as } n \rightarrow \infty .
$$

Consequently, the iterates $u_{n}(x)$ in (2.4) cannot converge to the unique solution $u(x) \equiv 0$ of $(2.1)$.

Thus, the direct iteration scheme (1.3) does not work for Example 2.1 if the initial state $u_{0}(x) \equiv C$ is not small. In contrast, the monotone iteration scheme (1.4) works for Example 2.1 for any $C \in \mathbb{R}$ if $u_{0}(x) \equiv C_{i}, i=1,2$, are properly chosen, because

(i) if $C_{1}>0$, then $\bar{u}(x) \equiv C_{1}$ satisfies

$$
\left\{\begin{array}{l}
\bar{u}^{\prime \prime}(x)-\bar{u}(x)^{3}=-C_{1}^{3}<0 \quad \text { on }(0,1) \\
\bar{u}(x)=C_{1}>0 \quad \text { at } \quad x=0,1
\end{array}\right.
$$

Therefore, $\bar{u}(x)$ is a supersolution, cf. (2.7).

(ii) If $C_{2}<0$, then $\bar{v}(x) \equiv C_{2}$ satisfies

$$
\left\{\begin{array}{l}
\bar{v}^{\prime \prime}(x)-\bar{v}(x)^{3}=-C_{2}^{3}>0 \quad \text { on }(0,1) \\
\bar{v}(x)=C_{2}<0 \quad \text { at } \quad x=0,1
\end{array}\right.
$$

Therefore $\bar{v}(x)$ is a subsolution, cf. (2.8).

If we choose $\lambda>0$ and $C \in \mathbb{R}$ such that

$$
\lambda>\max \left(3 C_{1}^{2}, 3 C_{2}^{2}\right), \quad C \in\left(C_{2}, C_{1}\right),
$$

then for the nonlinearity $F(x, u)=u^{3}$ in Example 2.1, we have

$$
\lambda-\frac{\partial F}{\partial u}=\lambda-3 u^{2}>0 \quad \forall u \in\left(C_{2}, C_{1}\right) .
$$

The monotone iteration scheme (1.4) will work for Example 2.1, by Theorem 2.2 below.

To make this paper sufficiently self-contained, in the following we state a version of the monotone iteration theorem. 
Theorem $2.2([2,12,17])$. Let $F(x, u)$ be $C^{1}$ with respect to $(x, u) \in \bar{\Omega} \times \mathbb{R}$. Consider the boundary value problem

$$
\begin{cases}\Delta u(x)-F(x, u(x))=0 & \text { on } \Omega, \\ B u(x)=g(x) & \text { on } \partial \Omega\end{cases}
$$

where $B$ is given by (1.2) with $\alpha \in C^{\infty}(\partial \Omega), \alpha(x) \geq 0$, and $\alpha(x) \not \equiv 0$ if $B u \neq u$ on $\partial \Omega$, and $g \in C^{2}(\partial \Omega)$. Let $\bar{u}, \bar{v} \in C^{2}(\bar{\Omega})$ satisfy $\bar{u} \geq \bar{v}$ as well as

$$
\begin{cases}\Delta \bar{u}(x)-F(x, \bar{u}(x)) \leq 0, & x \in \Omega, \\ B \bar{u}(x) \geq g(x), & x \in \partial \Omega,\end{cases}
$$

$$
\begin{cases}\Delta \bar{v}(x)-F(x, \bar{v}(x)) \geq 0, & x \in \Omega, \\ B \bar{v}(x) \leq g(x), & x \in \partial \Omega .\end{cases}
$$

(We call $\bar{u}$ and $\bar{v}$, respectively, a supersolution and a subsolution for satisfying (2.7) and (2.8).) Choose a number $\lambda>0$ such that

$$
\lambda-\frac{\partial F(x, u)}{\partial u}>0 \quad \forall(x, u) \in \bar{\Omega} \times[a, b], \quad a=\inf \bar{v}, \quad b=\sup \bar{u},
$$

and such that the operator $\left(\Delta-\lambda,\left.B\right|_{\partial \Omega}=0\right)$ has its spectrum strictly contained in the open left-half complex plane. Then the mapping

$$
\begin{array}{ll}
T: \phi \mapsto w, & w=T \phi, \\
\phi \in C^{2}(\bar{\Omega}), & \phi(x) \in[a, b] \quad \forall x \in \bar{\Omega},
\end{array}
$$

where $w(x)$ is the unique solution of the BVP

$$
\left\{\begin{array}{l}
\Delta w(x)-\lambda w(x)=-\lambda \phi(x)+F(x, \phi(x)), \quad x \in \Omega, \\
B w(x)=g(x), \quad x \in \partial \Omega,
\end{array}\right.
$$

is monotone, i.e., for any $\phi_{1}, \phi_{2}$ satisfying (2.11) and $\phi_{1} \leq \phi_{2}$, we have

$$
T \phi_{1}, T \phi_{2} \text { satisfy (2.11) and } T \phi_{1} \leq T \phi_{2} \text { on } \Omega \text {. }
$$

Consequently, by letting $F_{\lambda}(x, u) \equiv-\lambda u+F(x, u)$, the iterations

$$
\left\{\begin{array}{l}
u_{0}(x)=\bar{u}(x), \\
(\Delta-\lambda) u_{n+1}(x)=F_{\lambda}\left(x, u_{n}(x)\right), \quad x \in \Omega, \quad n=0,1,2, \ldots, \\
B u_{n+1}=g \quad \text { on } \partial \Omega
\end{array}\right.
$$


and

$$
\left\{\begin{array}{l}
v_{0}(x)=\bar{v}(x), \\
(\Delta-\lambda) v_{n+1}(x)=F_{\lambda}\left(x, v_{n}(x)\right), \quad x \in \Omega, \quad n=0,1,2, \ldots, \\
B v_{n+1}=g \quad \text { on } \partial \Omega,
\end{array}\right.
$$

yield iterates $u_{n}$ and $v_{n}$ satisfying

$$
\bar{v}=v_{0} \leq v_{1} \leq \cdots \leq v_{n} \leq \cdots \leq u_{n} \leq \cdots \leq u_{1} \leq u_{0}=\bar{u},
$$

so that the limits

$$
u_{\infty}(x) \equiv \lim _{n \rightarrow \infty} u_{n}(x)
$$

and

$$
v_{\infty}(x) \equiv \lim _{n \rightarrow \infty} v_{n}(x)
$$

exist in $C^{2}(\bar{\Omega})$. We have

(i) $v_{\infty} \leq u_{\infty}$ on $\bar{\Omega}$;

(ii) $v_{\infty}$ and $u_{\infty}$ are both stable solutions of (2.6);

(iii) If $v_{\infty} \not \equiv u_{\infty}$, then there exists an unstable solution $\phi \in C^{2}(\bar{\Omega})$ of (2.6) such that

$$
v_{\infty} \leq \phi \leq u_{\infty}
$$

Remark 2.3. The rate of convergence in (2.16) and (2.17) is not available in general. To make the numerical analysis possible in our subsequent development, obviously some additional working assumption is needed. Otherwise, numerical discretizations cause errors and make the computed solutions deviate outside the range of monotone interations (2.9). This will be formulated in the important $[\mathrm{H}]$ condition in $\S 4$.

Remark 2.4. (a) We note that there is a useful further formulation of the above theorem in the weak (i.e., distributional) sense.

(b) For simplicity of presentation, from now on, we will assume that $F, g, \bar{u}, \bar{v}$ and $\partial \Omega$ (consequently, also $u_{\infty}$ and $v_{\infty}$ ) are all $C^{\infty}(\bar{\Omega})$, considering that this is not the main issue here.

\section{Formulation of Boundary integral EQUATions BASED ON THE SIMPLE-LAYER REPRESENTATION}

Let $E(x)$ be the fundamental solution satisfying

$$
(\Delta-\lambda) E(x ; \lambda)=-\delta(x), \quad x \in \mathbb{R}^{N},
$$

where $\delta(x)$ is the Dirac delta function. It is known ([6, p. 341], e.g.) that

$$
E(x ; \lambda)=\frac{1}{4}(i)^{\frac{N}{2}}\left(\frac{\sqrt{\lambda}}{2 \pi|x|}\right)^{\frac{N-2}{2}} H_{\frac{N}{2}-1}^{(1)}(i \sqrt{\lambda}|x|), \quad N \geq 2,
$$


where $H_{\frac{N}{2}-1}^{(1)}(\cdot)$ is the Hankel function of order $\frac{N}{2}-1$ of the first kind. In particular, we have

$$
E(x ; \lambda)=\frac{1}{2 \pi} K_{0}(\sqrt{\lambda}|x|), \quad N=2,
$$

where $K_{0}$ is the Macdonald function of order 0 , and

$$
E(x ; \lambda)=\frac{e^{-\sqrt{\lambda}|x|}}{4 \pi|x|}, \quad N=3 .
$$

Let the assumptions of Theorem 2.2 be satisfied. We first consider the case when the boundary condition is Dirichlet:

$$
B u(x) \equiv u(x)=g(x), \quad \text { on } \partial \Omega,
$$

and defer the discussion of the Neumann and Robin boundary conditions to $\S 6$. For the boundary integral formulation of the monotone iteration

$$
\left\{\begin{array}{l}
(\Delta-\lambda) u_{n+1}(x)=F_{\lambda}\left(x, u_{n}(x)\right), \quad x \in \Omega, \\
u_{n+1}(x)=g(x), \quad x \in \partial \Omega,
\end{array}\right.
$$

the boundary integral equation by the standard direct formulation for the unknown $\partial u_{n+1} / \partial n$ is

$$
\begin{aligned}
\int_{\partial \Omega} E(x-y ; \lambda) \frac{\partial u_{n+1}(y)}{\partial n} d \sigma_{y}= & \frac{1}{2} g(x)+\int_{\partial \Omega} \frac{\partial E(x-y ; \lambda)}{\partial n_{y}} g(y) d \sigma_{y} \\
& -\int_{\Omega} E(x-y ; \lambda) F_{\lambda}\left(y, u_{n}(y)\right) d y, \quad x \in \partial \Omega,
\end{aligned}
$$

as given in Sakakihara [16, (3.3)]. Once $\partial u_{n+1} / \partial n$ is solved in (3.7), $u_{n+1}$ on the entire $\Omega$ can be obtained by quadratures on $\Omega$ and $\partial \Omega$.

The indirect formulation is based upon the following ansatz $([6,9,10])$

$$
\begin{aligned}
u_{n+1}(x) & =\left(\widetilde{S}_{\lambda} \eta_{n+1}\right)(x)-\widetilde{V}_{\lambda}\left(u_{n}\right)(x) \\
& \equiv \int_{\partial \Omega} E(x-y ; \lambda) \eta_{n+1}(y) d \sigma_{y}-\int_{\Omega} E(x-y ; \lambda) F_{\lambda}\left(y, u_{n}(y)\right) d y, \quad x \in \bar{\Omega},
\end{aligned}
$$

where $\eta_{n+1}(\cdot)$ is an unknown boundary layer density defined on $\partial \Omega$ to be solved by the BIE

$$
\int_{\partial \Omega} E(x-y ; \lambda) \eta_{n+1}(y) d \sigma_{y}=g(x)+\int_{\Omega} E(x-y ; \lambda) F_{\lambda}\left(y, u_{n}(y)\right) d y, \quad x \in \partial \Omega .
$$

Comparing (3.9) with (3.7), we see that a large amount of numerical quadrature (involved in the evaluation of $\int_{\partial \Omega} \frac{\partial E}{\partial n_{y}} g d \sigma_{y}$ ) is bypassed. This approach is more advantageous. 
Let $a, b \in \mathbb{R}, \lambda>0$ be chosen such that for any $\phi \in C^{\infty}(\bar{\Omega})$,

$$
\frac{\partial F(x, \phi(x))}{\partial u}-\lambda<0 \quad \forall \phi: a \leq \phi(x) \leq b, \quad x \in \bar{\Omega} .
$$

For any $\phi \in C^{\infty}(\bar{\Omega})$, the volume potential in (3.8) defines a continuous mapping

$$
\widetilde{V}_{\lambda}: C^{\infty}(\bar{\Omega}) \rightarrow C^{\infty}(\bar{\Omega}), \quad \widetilde{V}_{\lambda}(\phi)(x) \equiv \int_{\Omega} E(x-y ; \lambda) F_{\lambda}(y, \phi(y)) d y, \quad x \in \bar{\Omega},
$$

cf. [6, p. 216], e.g. By the trace theorem, we have

$$
\left.\widetilde{V}_{\lambda, \Gamma}(\phi) \equiv \widetilde{V}_{\lambda}(\phi)\right|_{\partial \Omega} \in C^{\infty}(\partial \Omega) .
$$

The trace of the simple-layer potential $\widetilde{S}_{\lambda}$ in (3.8) is denoted by $S_{\lambda}$ :

$$
\left.S_{\lambda} \eta \equiv \widetilde{S}_{\lambda} \eta\right|_{\partial \Omega} .
$$

It is well known that $S_{\lambda}$ has the following regularity:

$$
\left\{\begin{array}{l}
S_{\lambda}: H^{r}(\partial \Omega) \rightarrow H^{r+1}(\partial \Omega), \text { isomorphically, } \forall r \in \mathbb{R}, \\
S_{\lambda} \text { is a strongly elliptic pseudodifferential operator of order }-1 \text { on } \partial \Omega
\end{array}\right.
$$

Now define a mapping $\mathcal{T}$ on $C_{a, b}^{\infty}(\bar{\Omega}) \equiv\left\{\phi \in C^{\infty}(\bar{\Omega}) \mid a \leq \phi(x) \leq b\right.$ on $\left.\bar{\Omega}\right\}$ :

$$
\eta=\mathcal{T} \phi,
$$

where $\eta$ is a function defined on $\partial \Omega$ satisfying the BIE

$$
\int_{\partial \Omega} E(x-y ; \lambda) \eta(y) d \sigma_{y}=\int_{\Omega} E(x-y ; \lambda) F_{\lambda}(y, \phi(y)) d y, \quad x \in \partial \Omega .
$$

By (3.11), and (3.13)-(3.15), we have $\eta \in C^{\infty}(\partial \Omega)$, and that

$$
\mathcal{T}: C_{a, b}^{\infty}(\bar{\Omega}) \rightarrow C^{\infty}(\partial \Omega)
$$

is continuous.

We now establish the main theorem in this section.

Theorem 3.1. The mapping $\mathcal{T}$ in (3.14) is antimonotone, i.e.,

$$
\phi_{1}, \phi_{2} \in C_{a, b}^{\infty}(\bar{\Omega}), \quad \phi_{1}(x) \leq \phi_{2}(x) \quad \forall x \in \bar{\Omega}
$$

implies

$$
\mathcal{T} \phi_{1} \geq \mathcal{T} \phi_{2} \quad \text { on } \partial \Omega
$$

Proof. Let

$$
\eta_{i}=\mathcal{T} \phi_{i}, \quad i=1,2, \quad \text { on } \partial \Omega .
$$


Extend $\phi_{1}$ and $\phi_{2}$ to $\mathbb{R}^{N}$ by

$$
\tilde{\phi}_{i}(x)=\left\{\begin{array}{ll}
0, & x \in \Omega^{c} \equiv \mathbb{R}^{N} \backslash \bar{\Omega}, \\
\phi_{i}(x), & x \in \bar{\Omega},
\end{array} \quad i=1,2 .\right.
$$

Then through a localization procedure ([6, Chap. 6$])$ it is not difficult to show that

$$
\tilde{\phi}_{i} \in H^{\frac{1}{2}-\varepsilon}\left(\mathbb{R}^{N}\right), \quad \text { for any } \varepsilon>0, i=1,2 .
$$

From the definition of $\widetilde{S}_{\lambda} \eta$ in (3.8), by abuse of notation, extend $\widetilde{S}_{\lambda} \eta$ to all $\mathbb{R}^{N}$, i.e.,

$$
\left(\widetilde{S}_{\lambda} \eta\right)(x)=\int_{\partial \Omega} E(x-y ; \lambda) \eta(y) d \sigma_{y}, \quad x \in \mathbb{R}^{N} .
$$

We also extend $\widetilde{V}_{\lambda}$ to $\mathbb{R}^{N}$ by defining

$$
\left(\widetilde{V}_{\lambda} \tilde{\phi}\right)(x)=\int_{\Omega} E(x-y ; \lambda) \widetilde{F}_{\lambda}(y, \phi(y)) d y, \quad x \in \mathbb{R}^{N},
$$

where

$$
\widetilde{F}_{\lambda}(x, \phi(x)) \equiv \begin{cases}0, & \text { if } x \in \Omega^{c} \\ F_{\lambda}(x, \phi(x)), & \text { if } x \in \Omega .\end{cases}
$$

From (3.16), (3.19), (3.20), (3.22) and [6, Chap. 6], we have the regularities

$$
\eta_{i} \in C^{\infty}(\partial \Omega), \quad \tilde{V}_{\lambda} \tilde{\phi}_{i} \in H_{\mathrm{loc}}^{\frac{5}{2}-\varepsilon}\left(\mathbb{R}^{N}\right), \quad \text { for any } \varepsilon>0 .
$$

Now define

$$
\begin{aligned}
& w_{i}^{ \pm}(x)=\left(\widetilde{S}_{\lambda} \eta_{i}\right)(x)-\left(\widetilde{V}_{\lambda} \phi_{i}\right)(x), \quad i=1,2, \\
& \text { "+" if } x \in \Omega^{c}, \quad \text { "-" if } x \in \Omega .
\end{aligned}
$$

By (3.14) and (3.15), we have $\left.w_{i}^{-}\right|_{\partial \Omega} \equiv 0, i=1,2$. Also, on $\Omega$, we have

$$
(\Delta-\lambda) w_{i}^{-}(x)=F_{\lambda}\left(x, \phi_{i}(x)\right)=-\lambda \phi_{i}(x)+F\left(x, \phi_{i}(x)\right), \quad i=1,2 .
$$

Therefore, $w_{i}^{-}$is the (unique) solution of the BVP

$$
\left\{\begin{array}{llc}
(\Delta-\lambda) w_{i}^{-}(x)=-\lambda \phi_{i}(x)+F\left(x, \phi_{i}(x)\right) & \text { on } & \Omega, \\
w_{i}^{-}(x)=0 & \text { on } & \partial \Omega,
\end{array}\right\}, \quad i=1,2 .
$$

Similarly, from (3.15) and (3.22)-(3.24), $w_{i}^{+}$satisfies the exterior BVP

$$
\left\{\begin{array}{ll}
(\Delta-\lambda) w_{i}^{+}(x)=0 & \text { on } \quad \Omega^{c}, \\
w_{i}^{+}(x)=0 & \text { on } \quad \partial \Omega,
\end{array} \quad i=1,2 .\right.
$$


We now show that $w_{i}^{+}, i=1,2$, satisfying (3.24) and (3.26), are trivial. For $x \in \Omega^{c},|x|$ large, and $y \in \Omega$, we have

$$
\begin{aligned}
|x-y| & =\sqrt{|x|^{2}-2\langle x, y\rangle+|y|^{2}} \\
& =|x|\left[1-\frac{1}{2}\left(\frac{2\langle x, y\rangle-|y|^{2}}{|x|^{2}}\right)+\frac{\frac{1}{2}\left(-\frac{1}{2}\right)}{1 \cdot 2}\left(\frac{-2\langle x, y\rangle+|y|^{2}}{|x|^{2}}\right)^{2} \pm \cdots\right] .
\end{aligned}
$$

The fundamental solution $E(x ; \lambda)$ in $(3.2)$ is known to have fast decay for $|x|$ large when $\lambda>0$. For example, when $N=3$, this is obvious from (3.4). For $N=2$, we have [1]

$$
\begin{aligned}
E(x, \lambda) & =\frac{1}{2 \pi} K_{0}(\sqrt{\lambda}|x|) \\
& \sim \frac{1}{2} \sqrt{\frac{1}{2 \sqrt{\lambda}|x|}} e^{-\sqrt{\lambda}|x|}\left\{1-\frac{1}{8 \sqrt{\lambda}|x|} \pm \cdots\right\} .
\end{aligned}
$$

Using (3.27) and (3.28) in (3.24), we obtain

$$
w_{i}^{+}(x)=\left\{\begin{aligned}
\frac{1}{2} \lambda^{-\frac{1}{4}} \sqrt{\frac{1}{2|x|}}\left[1+\mathcal{O}\left(|x|^{-\frac{1}{2}}\right)\right]\left\{\int_{\partial \Omega} e^{-\sqrt{\lambda}|x-y|} \eta(y) d \sigma_{y}\right. & \\
& \left.-\int_{\Omega} e^{-\sqrt{\lambda}|x-y|} F_{\lambda}\left(y, \phi_{i}(y)\right) d y\right\}, \quad N=2 ; \\
\frac{1}{4 \pi} \frac{1}{|x|}\left[1+\mathcal{O}\left(|x|^{-1}\right)\right] & \left\{\int_{\partial \Omega} e^{-\sqrt{\lambda}|x-y|} \eta(y) d \sigma_{y}\right. \\
& \left.-\int_{\Omega} e^{-\sqrt{\lambda}|x-y|} F_{\lambda}\left(y, \phi_{i}(y)\right) d y\right\}, \quad N=3 .
\end{aligned}\right.
$$

For space dimension $N>3$, similar expressions can be obtained. Note that each integral inside the parentheses above decay with an exponential rate $e^{-\sqrt{\lambda}|x|}$ for $|x|$ large.

Let

$$
\Omega_{R}^{c}=\Omega^{c} \cap\left\{x \in \mathbb{R}^{N}|| x \mid<R\right\}, \text { for large } R>0 .
$$

From (3.26), we obtain

$$
\begin{aligned}
0 & =\int_{\Omega^{c}}\left[(\Delta-\lambda) w_{i}^{+}(x)\right] w_{i}^{+}(x) d x \\
& =\lim _{R \rightarrow \infty}\left[\int_{\partial \Omega_{R}^{c}} \frac{\partial w_{i}^{+}(x)}{\partial n} w_{i}^{+}(x) d \sigma-\int_{\Omega_{R}^{c}}\left(\left|\nabla w_{i}^{+}(x)\right|^{2}+\lambda\left|w_{i}^{+}(x)\right|^{2}\right) d x\right] \\
& =-\int_{\Omega^{c}}\left(\left|\nabla w_{i}^{+}(x)\right|^{2}+\lambda\left|w_{i}^{+}(x)\right|^{2}\right) d x,
\end{aligned}
$$

where the limit of the boundary integral over $\partial \Omega_{R}^{c}$ tends to 0 as $R \rightarrow \infty$ because

$$
\begin{aligned}
\int_{\partial \Omega_{R}^{c}} \frac{\partial w_{i}^{+}(x)}{\partial n} w_{i}^{+}(x) d \sigma & =\int_{\partial \Omega^{c}} \frac{\partial w_{i}^{+}(x)}{\partial n} w_{i}^{+}(x) d \sigma+\int_{|x|=R} \frac{\partial w_{i}^{+}(x)}{\partial r} w_{i}^{+}(x) d \sigma \\
& =\int_{|x|=R} \frac{\partial w_{i}^{+}(x)}{\partial r} w_{i}^{+}(x) d \sigma \quad(r=|x|),
\end{aligned}
$$


and the above integral tends to zero as $R \rightarrow \infty$ by (3.29). Hence $w_{i}^{+}(x) \equiv 0$ on $\Omega^{c}$, $i=1,2$. Define

$$
w(x)= \begin{cases}w_{2}^{+}(x)-w_{1}^{+}(x), & x \in \Omega^{c} \\ w_{2}^{-}(x)-w_{1}^{-}(x), & x \in \Omega\end{cases}
$$

Then

$$
w(x) \equiv 0 \quad \text { for } \quad x \in \Omega^{c} .
$$

On $\Omega$, the function $w$ satisfies

$$
\begin{aligned}
(\Delta-\lambda) w(x) & =-\lambda\left[\phi_{2}(x)-\phi_{1}(x)\right]+\left[F\left(x, \phi_{2}(x)\right)-F\left(x, \phi_{1}(x)\right)\right] \\
& =\left[\phi_{2}(x)-\phi_{1}(x)\right] \cdot\left[-\lambda+\frac{F\left(x, \phi_{2}(x)\right)-F\left(x, \phi_{1}(x)\right)}{\phi_{2}(x)-\phi_{1}(x)}\right] \\
& \leq 0 \quad \text { by }(3.12),
\end{aligned}
$$

and on $\partial \Omega$, it satisfies $w \equiv 0$. Thus,

$$
\begin{cases}(\Delta-\lambda) w \leq 0 & \text { on } \quad \Omega \\ \left.w\right|_{\partial \Omega}=0 & \text { on } \quad \partial \Omega .\end{cases}
$$

If $w(x)<0$ for some $x \in \Omega$, then

$$
\min _{x \in \bar{\Omega}} w(x)<0 .
$$

Assume that

$$
w\left(x_{0}\right)=\min _{x \in \bar{\Omega}} w(x) .
$$

Then $x_{0} \notin \Omega$ by Hopf's boundary point lemma [13]. Thus $x_{0} \in \partial \Omega$. But

(3.35) $\quad 0>w\left(x_{0}\right)=0, \quad$ by the boundary condition in (3.33), a contradiction.

Therefore,

$$
w(x) \geq 0 \quad \text { on } \Omega .
$$

Let $\widetilde{V}_{\lambda} \tilde{\phi}_{i}$ be the volume potential in (3.23). Since $\widetilde{F}_{\lambda}\left(\cdot, \phi_{i}(\cdot)\right) \in H^{\frac{1}{2}-\varepsilon}\left(\mathbb{R}^{N}\right)$ (just as in (3.20)), by elliptic regularity we have

$$
\tilde{V}_{\lambda} \tilde{\phi}_{i} \in H^{\frac{5}{2}-\varepsilon}\left(\mathbb{R}^{N}\right), \quad i=1,2, \text { for any } \varepsilon>0 .
$$

Further, let

$$
\begin{aligned}
\left(\widetilde{V}_{\lambda} \tilde{\phi}_{i}\right)^{+} & =\widetilde{V}_{\lambda} \tilde{\phi}_{i}(x) & \text { if } & x \in \Omega^{c}, \\
\left(\widetilde{V}_{\lambda} \tilde{\phi}_{i}\right)^{-}(x) & =\widetilde{V}_{\lambda} \tilde{\phi}_{i}(x) & \text { if } & x \in \Omega .
\end{aligned}
$$

Then the trace theorem gives

$$
\frac{\partial}{\partial n}\left(\widetilde{V}_{\lambda} \tilde{\phi}_{i}\right)^{ \pm} \in H^{1-\varepsilon}(\partial \Omega), \quad \text { for any } \quad 0<\varepsilon<1 .
$$


Choose any $\varepsilon$ : $0<\varepsilon<1$. Then by the continuity of the normal trace operation we have

$$
\frac{\partial}{\partial n}\left(\widetilde{V}_{\lambda} \tilde{\phi}_{i}\right)^{+}-\frac{\partial}{\partial n}\left(\widetilde{V}_{\lambda} \tilde{\phi}_{i}\right)^{-}=\frac{\partial}{\partial n} \widetilde{V}_{\lambda} \tilde{\phi}_{i}-\frac{\partial}{\partial n} \widetilde{V}_{\lambda} \tilde{\phi}_{i}=0 \quad \text { on } \partial \Omega, i=1,2
$$

Also, let

$$
\begin{gathered}
\left(\widetilde{S}_{\lambda} \eta_{i}\right)^{ \pm}(x)=\int_{\partial \Omega} E(x-\xi ; \lambda) \eta_{i}(\xi) d \sigma_{\xi}, \quad i=1,2, \\
\text { "+" if } x \in \Omega^{c}, \quad \text { "_" if } x \in \Omega .
\end{gathered}
$$

Then, on $\partial \Omega$,

$$
\begin{aligned}
\frac{\partial}{\partial n}\left[w^{+}(x)-w^{-}(x)\right] & =-\frac{\partial}{\partial n} w^{-}(x) \quad(\text { by }(3.31)) \\
& =\lim _{h \downarrow 0} \frac{w^{-}(x-n h)-w^{-}(x)}{h} \\
& =\lim _{h \downarrow 0} \frac{w^{-}(x-n h)}{h} \quad(x \in \partial \Omega) \\
& \geq 0 \text { by }(3.36) .
\end{aligned}
$$

But from (3.38), we also have

$$
\begin{aligned}
\frac{\partial}{\partial n}\left[w^{+}\right. & \left.(x)-w^{-}(x)\right] \\
= & \frac{\partial}{\partial n}\left\{\left[\left(\left(\widetilde{S}_{\lambda} \eta_{2}\right)^{+}(x)-\left(\left(\widetilde{V}_{\lambda} \tilde{\phi}_{2}\right)^{+}(x)\right)-\left(\left(\widetilde{S}_{\lambda} \eta_{1}\right)^{+}(x)-\left(\widetilde{V}_{\lambda} \tilde{\phi}_{1}\right)^{+}(x)\right)\right]\right.\right. \\
& \left.-\left[\left(\left(\widetilde{S}_{\lambda} \eta_{2}\right)^{-}(x)-\left(\widetilde{V}_{\lambda} \tilde{\phi}_{2}\right)^{-}(x)\right)-\left(\left(\widetilde{S}_{\lambda} \eta_{1}\right)^{-}(x)-\left(\widetilde{V}_{\lambda} \phi_{1}\right)^{-}(x)\right)\right]\right\} \\
= & \frac{\partial}{\partial n}\left\{\left[\left(\widetilde{S}_{\lambda} \eta_{2}\right)^{+}(x)-\left(\widetilde{S}_{\lambda} \eta_{1}\right)^{+}(x)\right]-\left[\left(\widetilde{S}_{\lambda} \eta_{2}\right)^{-}(x)-\left(\widetilde{S}_{\lambda} \eta_{1}\right)^{-}(x)\right]\right\} \\
= & -\eta_{2}(x)-\left(-\eta_{1}(x)\right) \quad \text { (by }[6, \text { Corollary 6.5.1]) } \\
= & \eta_{1}(x)-\eta_{2}(x) \geq 0 \quad \text { by }(3.40) . \quad \square
\end{aligned}
$$

Hence the proof is complete.

Some numerical evidence of Theorem 3.1 is provided in Fig. 7.2.

Corollary 3.2. Let $g \in C^{\infty}(\partial \Omega)$ be given. Let (3.10) be satisfied. Define

$$
\mathcal{T}_{g}: C_{a, b}^{\infty}(\bar{\Omega}) \rightarrow C^{\infty}(\partial \Omega), \quad \mathcal{T}_{g} \phi=\eta
$$

where $\eta$ is the solution of the BIE

$$
\int_{\partial \Omega} E(x-y ; \lambda) \eta_{n+1}(y) d \sigma_{y}=g(x)+\int_{\Omega} E(x-y ; \lambda) F_{\lambda}(y, \phi(y)) d y, \quad x \in \partial \Omega
$$

Then $\mathcal{T}_{g}$ is also antimonotone. 
Corollary 3.3. Let the assumptions of Theorem 2.1 be satisfied. Let the monotone iterations

$$
\left\{\begin{array}{l}
u_{0}(x)=\bar{u}(x) \in C^{\infty}(\bar{\Omega}), \\
(\Delta-\lambda) u_{n+1}(x)=F_{\lambda}\left(x, u_{n}(x)\right), \quad x \in \Omega, \\
u_{n+1}(x)=g(x), \quad x \in \partial \Omega,
\end{array}\right.
$$

and

$$
\left\{\begin{array}{l}
v_{0}(x)=\bar{v}(x) \in C^{\infty}(\bar{\Omega}), \\
(\Delta-\lambda) v_{n+1}(x)=F_{\lambda}\left(x, v_{n}(x)\right), \quad x \in \Omega, \\
v_{n+1}(x)=g(x), \quad x \in \partial \Omega,
\end{array}\right.
$$

have iterative solutions $u_{n+1}$ and $v_{n+1}$ represented, for $n=0,1,2, \ldots$, as

$$
u_{n+1}(x)=\int_{\partial \Omega} E(x-y ; \lambda) \mu_{n+1}(y) d \sigma_{y}-\int_{\Omega} E(x-y ; \lambda) F_{\lambda}\left(y, u_{n}(y)\right) d y, x \in \bar{\Omega},
$$

$$
v_{n+1}(x)=\int_{\partial \Omega} E(x-y ; \lambda) \nu_{n+1}(y) d \sigma_{y}-\int_{\Omega} E(x-y ; \lambda) F_{\lambda}\left(y, v_{n}(y)\right) d y, x \in \bar{\Omega},
$$

where the simple-layer densities $\mu_{n+1}(\cdot)$ and $\nu_{n+1}(\cdot)$ are determined from

$$
\mu_{n+1}=\mathcal{T}_{g} u_{n}, \quad \nu_{n+1}=\mathcal{T}_{g} v_{n}, \quad n=0,1,2, \ldots .
$$

Then

$$
\mu_{\infty}=\lim _{n \rightarrow \infty} \mu_{n}, \quad \nu_{\infty}=\lim _{n \rightarrow \infty} \nu_{n}
$$

exist in $C^{\infty}(\partial \Omega)$ such that

$$
\mu_{1} \leq \mu_{2} \leq \cdots \leq \mu_{n} \leq \cdots \rightarrow \mu_{\infty} \leq \nu_{\infty} \leftarrow \leq \nu_{n} \leq \cdots \leq \nu_{2} \leq \nu_{1}
$$

As in Remark 2.4, we again note that the rate of convergence of (3.48) is not available in general.

\section{Error estimates for the Galerkin boundary Element scheme}

We first recall some basic regularity properties of the operators corresponding to the simple-layer and volume potentials. For $\phi \in C^{\infty}(\bar{\Omega})$, let $\mathcal{V}_{\lambda} \phi$ denote the volume potential defined by

$$
\left(\mathcal{V}_{\lambda} \phi\right)(x)=\int_{\Omega} E(x-y ; \lambda) \phi(y) d y, \quad x \in \bar{\Omega} .
$$

Then it is known (cf. [6, Theorem 6.3.1], e.g.) that $\mathcal{V}_{\lambda}$ can be extended to be an operator such that

$$
\left\{\begin{array}{l}
\mathcal{V}_{\lambda}: H^{r}(\Omega) \rightarrow H^{r+2}(\Omega) \quad \text { continuous, } \quad \forall r \geq-1 . \\
\left\|\mathcal{V}_{\lambda} \phi\right\|_{H^{r+2}(\Omega)} \leq \bar{C}_{r}\|\phi\|_{H^{r}(\Omega)}, \quad \forall \phi \in H^{r}(\Omega), \quad r \geq-1
\end{array}\right.
$$


for a constant $\bar{C}_{r}>0$ independent of $\phi$. The trace of (4.1) induces an operator $\mathcal{V}_{\lambda, \Gamma}$ defined by

$$
\left(\mathcal{V}_{\lambda, \Gamma} \phi\right)(x) \equiv\left(\mathcal{V}_{\lambda} \phi\right)(x), \quad x \in \partial \Omega
$$

it satisfies

$$
\left\{\begin{array}{l}
\mathcal{V}_{\lambda, \Gamma}: H^{r}(\Omega) \rightarrow H^{r+\frac{3}{2}}(\partial \Omega) \quad \text { continuous, } \quad \forall r \geq-1 \\
\text { there exists } \bar{C}_{r}>0 \text { such that } \\
\left\|\mathcal{V}_{\lambda, \Gamma} \phi\right\|_{H^{r+\frac{3}{2}(\partial \Omega)}} \leq \overline{\bar{C}}_{r}\|\phi\|_{H^{r}(\Omega)}, \quad \forall \phi \in H^{r}(\Omega), r \geq-1
\end{array}\right.
$$

Note the relationship

$$
\widetilde{V}_{\lambda} \phi=\mathcal{V}_{\lambda} F(\cdot, \phi(\cdot))
$$

from (3.8).

Let the simple-layer potential $\widetilde{S}_{\lambda}$ be as defined in (3.8), and let $S_{\lambda}$ be the corresponding trace as given in (3.12). It is known ([6, Chap. 6]) that

$$
\left\{\begin{array}{l}
\widetilde{S}_{\lambda}: H^{r}(\partial \Omega) \rightarrow H^{r+\frac{3}{2}}(\Omega) \quad \text { continuous, } \quad \forall r \in \mathbb{R}, \\
\text { there exists } \widetilde{C}_{r}>0, \text { such that } \\
\left\|\widetilde{S}_{\lambda} \eta\right\|_{H^{r+\frac{3}{2}(\Omega)}} \leq \widetilde{C}_{r}\|\eta\|_{H^{r}(\partial \Omega)}, \quad \forall \eta \in H^{r}(\partial \Omega) .
\end{array}\right.
$$

Also, from (3.13), for each $r \in \mathbb{R}$, there exists a $\widetilde{\widetilde{C}}_{r}>0$ such that

$$
\widetilde{\widetilde{C}}_{r}^{-1}\|\eta\|_{H^{r}(\partial \Omega)} \leq\left\|S_{\lambda} \eta\right\|_{H^{r+1}(\partial \Omega)} \leq \widetilde{\widetilde{C}}_{r}\|\eta\|_{H^{r}(\partial \Omega)}, \quad \forall \eta \in H^{r}(\partial \Omega) .
$$

Let $\left\{\mathcal{S}_{h} \mid 0<h \leq h_{0}\right\}$ be a 1-parameter family of finite-dimensional boundary element approximation spaces that form an $(\ell, m)$-system $S_{h}^{\ell, m}(\partial \Omega)$, with $\ell, m \in$ $\mathbb{Z}^{+} \equiv\{0,1,2, \ldots \ldots\}, \ell \geq m+1$, on $\partial \Omega$, in the sense of Babuška and Aziz [4], satisfying

\section{(1) Approximation property:}

For each $\phi \in H^{t}(\partial \Omega)$, there exists a $\phi_{h} \in \mathcal{S}_{h}$ such that

$$
\left\|\phi-\phi_{h}\right\|_{H^{s}(\partial \Omega)} \leq C_{t, s} h^{t-s}\|\phi\|_{H^{t}(\partial \Omega)}, \quad \forall h: 0<h \leq h_{0},
$$

where $-\ell \leq s \leq t \leq \ell ;|s|,|t| \leq m$, and $C_{t, s}$ is a constant independent of $h$ and $\phi$.

(2) Inverse property:

There exists a constant $M_{s, t}>0$ such that

$$
\left\|\phi_{h}\right\|_{H^{t}(\partial \Omega)} \leq M_{s, t} h^{s-t}\left\|\phi_{h}\right\|_{H^{s}(\partial \Omega)}, \quad \forall \phi_{h} \in \mathcal{S}_{h}, \quad \forall h: 0<h \leq h_{0},
$$

where $s \leq t$, and $|s|,|t| \leq m$.

We now implement the monotone iteration scheme according to Corollary 3.3. For definiteness, we will only discretize the (supersolution) systems (3.47) and (3.49). The subsolution systems (3.43) and (3.46) are analogous and thus can 
be handled in a nearly identical way. The Galerkin boundary element method can be formulated as follows:

(i) Set

$$
u_{h}^{0}(x)=\bar{u}(x), x \in \bar{\Omega}, \text { cf. the first equation in(3.43). }
$$

(ii) For each $n=0,1,2, \ldots$, and $h: 0<h \leq h_{0}$, find $\eta_{h}^{n+1} \in \mathcal{S}_{h}$ such that

$$
\left\langle S_{\lambda} \eta_{h}^{n+1}, \phi_{h}\right\rangle=\left\langle g+\mathcal{V}_{\lambda, \Gamma} F\left(\cdot, u_{h}^{n}\right), \phi_{h}\right\rangle, \quad \forall \phi_{h} \in \mathcal{S}_{h},
$$

where $\langle$,$\rangle signifies the L^{2}(\partial \Omega)$ inner product.

(iii) Define

$$
u_{h}^{n+1}(x)=\left(\widetilde{S}_{\lambda} \eta_{h}^{n+1}\right)(x)-\left(\mathcal{V}_{\lambda} F\left(\cdot, u_{h}^{n}\right)\right)(x), \quad x \in \bar{\Omega}, \quad n=0,1,2, \ldots .
$$

Note that (4.11) and (4.12) correspond, respectively, to the discretization of (3.42) and (3.45). We also see that in (4.11) and (4.12), quadrature evaluations are involved in the determination of $\mathcal{V}_{\lambda} F\left(\cdot, u_{h}^{n}\right)$ and $\mathcal{V}_{\lambda, \Gamma} F\left(\cdot, u_{h}^{n}\right)$. Since, in principle, these quadrature evaluations may be carried out to as high an accuracy as desired, we assume that $\mathcal{V}_{\lambda} F\left(\cdot, u_{h}^{n}\right)$ and $\mathcal{V}_{\lambda, \Gamma} F\left(\cdot, u_{h}^{n}\right)$ are exact (although in practice, these quadrature evaluations are rather tedious because the integration is carried out on the domain, and numerical errors are involved). From now on, without further mention, we also maintain all the assumptions and notation used in Theorem 2.2.

Our main objective is to estimate $\left\|u_{\infty}-u_{h}^{n}\right\|_{H^{r}(\Omega)}$, where $u_{\infty}$ is a stable solution as announced in Theorem 2.2(ii). The following working assumption is crucial; it enables us to obtain rates of convergence:

$[\mathrm{H}] \quad\left\{\begin{array}{l}\text { There exist constants } \gamma_{1} \text { and } \gamma_{2} \text { such that } \\ -\lambda_{1}<\gamma_{1} \leq \frac{\partial F(x, u)}{\partial u} \leq \gamma_{2}, \quad \forall(x, u) \in \bar{Q}, \quad \bar{Q} \equiv \bar{\Omega} \times[a, b], \\ \text { where } \lambda_{1}>0 \text { is the smallest eigenvalue of }-\Delta \text { on } \Omega \text { subject } \\ \text { to the homogeneous Dirichlet boundary condition. }\end{array}\right.$

Because $\frac{\partial F}{\partial u}$ is continuous, it easily follows from $[\mathrm{H}]$ that for a sufficiently small $\delta>0$, there exist $\tilde{\gamma}_{1}$ and $\tilde{\gamma}_{2}$ such that

$$
-\lambda_{1}<\tilde{\gamma}_{1} \leq \frac{\partial F(x, u)}{\partial u} \leq \tilde{\gamma}_{2}, \quad \forall(x, u) \in \bar{Q}_{\delta}, \quad \bar{Q}_{\delta} \equiv \bar{\Omega} \times[a-\delta, b+\delta] .
$$

This small $\delta$ will provide the "breathing room" to accommodate the errors in the numerical operation for the iterates to stay within the range of monotone iteration.

Lemma 4.1. Let $u_{n+1}$ be iterated according to (3.43) and let $u_{\infty}$ be the limit as in (3.48). Denote $e^{n}=u_{\infty}-u_{n}$. Then we have

$$
\left\|e^{n+1}\right\|_{L^{2}(\Omega)} \leq \alpha_{\lambda}^{n+1}\left\|e^{0}\right\|_{L^{2}(\Omega)}, \quad n=0,1,2, \ldots,
$$

where

$$
0<\alpha_{\lambda} \equiv \frac{\lambda-\tilde{\gamma}_{1}}{\lambda+\lambda_{1}}<1
$$


Proof. Since $u_{\infty}$ and $u_{n+1}$ satisfy, respectively, (2.6) (with $B u \equiv u$ ) and (3.43), we obtain by subtraction

$$
\begin{cases}(\Delta-\lambda) e^{n+1}(x)=F_{\lambda}\left(x, u_{\infty}(x)\right)-F_{\lambda}\left(x, u_{n}(x)\right), & x \in \Omega \\ e^{n+1}(x)=0, & x \in \partial \Omega .\end{cases}
$$

Multiplying the above by $-e^{n+1}$, integrating by parts and using $\lambda_{1}\left\|e^{n+1}\right\|^{2} \leq$ $\left\|\nabla e^{n+1}\right\|^{2}$, we get

$$
\left\|e^{n+1}\right\| \leq \frac{1}{\lambda+\lambda_{1}}\left\|F_{\lambda}\left(\cdot, u_{\infty}\right)-F_{\lambda}\left(\cdot, u_{n}\right)\right\|
$$

where all the norms without subscripts are $L^{2}(\Omega)$. Let

$$
M_{\lambda} \equiv \sup _{(x, u) \in \bar{Q}_{\delta}}\left[\lambda-\frac{\partial F(x, u)}{\partial u}\right] .
$$

Then

$$
0<M_{\lambda}<\lambda-\tilde{\gamma}_{1}
$$

(Actually, $\tilde{\gamma}_{1}$ can be taken to be $\gamma_{1}$ here.) We get

$$
\begin{aligned}
\left\|F_{\lambda}\left(\cdot, u_{\infty}\right)-F_{\lambda}\left(\cdot, u_{n}\right)\right\| & =\left\|-\left[\lambda-\frac{F\left(\cdot, u_{\infty}\right)-F\left(\cdot, u_{n}\right)}{u_{\infty}-u_{n}}\right]\left(u_{\infty}-u_{n}\right)\right\| \\
& \leq M_{\lambda}\left\|u_{\infty}-u_{n}\right\| .
\end{aligned}
$$

Substituting (4.20) into (4.17), we obtain

$$
\left\|e^{n+1}\right\| \leq \frac{M_{\lambda}}{\lambda+\lambda_{1}}\left\|e^{n}\right\| \leq \alpha_{\lambda}\left\|e^{n}\right\|, \quad \alpha_{\lambda} \equiv \frac{\lambda-\tilde{\gamma}_{1}}{\lambda+\lambda_{1}}<1 .
$$

Hence (4.14) follows.

Lemma 4.2. Let $\eta_{h}^{n+1}$ and $u_{h}^{n+1}$ be defined as in (4.11) and (4.12) for $n=$ $0,1,2, \ldots$ Let $\zeta_{h}^{n+1}$, defined on $\partial \Omega$, be the (unique) solution of the BIE

$$
\left(S_{\lambda} \zeta_{h}^{n+1}\right)(x)=g(x)+\mathcal{V}_{\lambda, \Gamma} F\left(x, u_{h}^{n}(x)\right), \quad x \in \partial \Omega .
$$

Then there exists $\widetilde{C}_{t, s}>0$, depending only on $s$ and $t$, such that

$$
\left\|\eta_{h}^{n+1}-\zeta_{h}^{n+1}\right\|_{H^{s}(\partial \Omega)} \leq \widetilde{C}_{t, s} h^{t-s}\left\|\zeta_{h}^{n+1}\right\|_{H^{t}(\partial \Omega)},
$$

where $m \leq \ell-1,-(\ell+1) \leq s \leq t \leq \ell, s \leq m,-\ell \leq-\frac{1}{2} \leq m$ and $-m \leq-\frac{1}{2} \leq t$.

Proof. Since $S_{\lambda}$ satisfies (4.7), according to Hsiao and Wendland [10], the order of $S_{\lambda}$ is $2 \alpha=-1$, i.e., $\alpha=-\frac{1}{2}$. Invoking [10, Corollary 2.1], we obtain the conclusion. 
Lemma 4.3. Let $N=2$ or 3 , and let

$$
\varepsilon_{0}(N)= \begin{cases}0, & \text { if } N=2, \\ \text { an arbitrarily small positive number, } & \text { if } N=3 .\end{cases}
$$

Then for $\ell \geq 2$ and $m \geq 1$, there exist positive constants $K_{\varepsilon_{0}(N)}$ and $K$ such that

$$
\begin{aligned}
\left\|\eta_{h}^{n+1}-\zeta_{h}^{n+1}\right\|_{H^{\varepsilon_{0}(N)}(\partial \Omega)} \leq K_{\varepsilon_{0}(N)} h^{\frac{1}{2}-\varepsilon_{0}(N)}\left[\left\|u_{\infty}\right\|_{H^{2}(\Omega)}+M_{\lambda} \alpha_{\lambda}^{n}\left\|e^{0}\right\|_{L^{2}(\Omega)}\right. \\
\left.+\left\|F_{\lambda}\left(\cdot, u_{h}^{n}\right)-F_{\lambda}\left(\cdot, u_{n}\right)\right\|_{L^{2}(\Omega)}\right],
\end{aligned}
$$

$$
\begin{array}{r}
\left\|\eta_{h}^{n+1}-\zeta_{h}^{n+1}\right\|_{H^{-\frac{3}{2}}(\partial \Omega)} \leq K h^{2}\left[\left\|u_{\infty}\right\|_{H^{2}(\Omega)}+M_{\lambda} \alpha_{\lambda}^{n}\left\|e^{0}\right\|_{L^{2}(\Omega)}\right. \\
\left.+\left\|F_{\lambda}\left(\cdot, u_{h}^{n}\right)-F_{\lambda}\left(\cdot, u_{n}\right)\right\|_{L^{2}(\Omega)}\right] \\
\forall n=0,1,2, \ldots, 0<h \leq h_{0} .
\end{array}
$$

Proof. We have from (4.22) and property (4.7) of $S_{\lambda}$,

$$
\begin{aligned}
& \left\|\eta_{h}^{n+1}-\zeta_{h}^{n+1}\right\|_{H^{\varepsilon_{0}(N)}(\partial \Omega)} \leq \widetilde{C}_{\frac{1}{2}, \varepsilon_{0}(N)} h^{\frac{1}{2}-\varepsilon_{0}(N)} \cdot \widetilde{\widetilde{C}}_{\frac{1}{2}}\left\|S_{\lambda} \zeta_{h}^{n+1}\right\|_{H^{\frac{3}{2}}(\partial \Omega)} \\
& \left.\leq\left(\widetilde{C}_{\frac{1}{2}, \varepsilon_{0}(N)} \widetilde{\widetilde{C}}_{\frac{1}{2}}\right) h^{\frac{1}{2}-\varepsilon_{0}(N)}\left[\|g\|_{H^{\frac{3}{2}}(\partial \Omega)}+\left\|\mathcal{V}_{\lambda, \Gamma} F_{\lambda}\left(\cdot, u_{h}^{n}\right)\right\|_{H^{\frac{3}{2}}(\partial \Omega)}\right] \quad \text { (by }(4.21)\right) \\
& \leq\left(\widetilde{C}_{\frac{1}{2}, \varepsilon_{0}(N)} \widetilde{\widetilde{C}}_{\frac{1}{2}}\right) h^{\frac{1}{2}-\varepsilon_{0}(N)}\left[\|g\|_{H^{\frac{3}{2}}(\partial \Omega)}+\overline{\bar{C}}_{0}\left\|F_{\lambda}\left(\cdot, u_{h}^{n}\right)\right\|_{L^{2}(\Omega)}\right] \quad \text { (by (4.4)) } \\
& \leq\left(\widetilde{C}_{\frac{1}{2}, \varepsilon_{0}(N)} \widetilde{\widetilde{C}}_{\frac{1}{2}}\right) h^{\frac{1}{2}-\varepsilon_{0}(N)}\left[\|g\|_{H^{\frac{3}{2}}(\partial \Omega)}+\overline{\bar{C}}_{0}\left(\left\|F_{\lambda}\left(\cdot, u_{h}^{n}\right)-F_{\lambda}\left(\cdot, u_{n}\right)\right\|_{L^{2}(\Omega)}\right.\right. \\
& \left.+\left\|F_{\lambda}\left(\cdot, u_{n}\right)-F_{\lambda}\left(\cdot, u_{\infty}\right)\right\|_{L^{2}(\Omega)}+\left\|F_{\lambda}\left(\cdot, u_{\infty}\right)\right\|_{L^{2}(\Omega)}\right] \\
& \leq\left(\widetilde{C}_{\frac{1}{2}, \varepsilon_{0}(N)} \widetilde{\widetilde{C}}_{\frac{1}{2}}\right) h^{\frac{1}{2}-\varepsilon_{0}(N)}\left[C\left\|u_{\infty}\right\|_{H^{2}(\Omega)}+\overline{\bar{C}}_{0} M_{\lambda} \alpha_{\lambda}^{n}\left\|e^{0}\right\|_{L^{2}(\Omega)}\right. \\
& \left.+\overline{\bar{C}}_{0}\left\|F_{\lambda}\left(\cdot, u_{h}^{n}\right)-F_{\lambda}\left(\cdot, u_{n}\right)\right\|_{L^{2}(\Omega)}\right],
\end{aligned}
$$

where in the last inequality we have utilized the properties that $u_{\infty}$ satisfies (2.6), the trace theorem, (4.14) and (4.20). Therefore, (4.24) has been established. From (4.22), we have

$$
\begin{aligned}
\left\|\eta_{h}^{n+1}-\zeta_{h}^{n+1}\right\|_{H^{-\frac{3}{2}}(\partial \Omega)} & \leq \widetilde{C}_{\frac{1}{2},-\frac{3}{2}} h^{2}\left\|\zeta_{h}^{n+1}\right\|_{H^{\frac{1}{2}}(\partial \Omega)} \\
& \leq \widetilde{C}_{\frac{1}{2},-\frac{3}{2}} h^{2} \cdot \widetilde{C}_{\frac{1}{2}}\left\|S_{\lambda} \zeta_{h}^{n+1}\right\|_{H^{\frac{3}{2}}(\partial \Omega)} .
\end{aligned}
$$

The rest is the same as in (4.26).

Remark 4.4. Lemma 4.3 remains valid even if $\varepsilon_{0}(N)=0$ when $N=3$, where the constant $K_{\varepsilon_{0}(N)}$ remains bounded as $\varepsilon_{0}(N) \downarrow 0$. But in the discussions henceforth we will only utilize the part when $\varepsilon_{0}(N)$ is positive yet nonvanishingly small.

From (4.21), we now define

$$
w_{h}^{n+1}(x)=\left(\widetilde{S}_{\lambda} \zeta_{h}^{n+1}\right)(x)-\left(\mathcal{V}_{\lambda} F\left(\cdot, u_{h}^{n}\right)\right)(x), \quad x \in \Omega, n=0,1,2, \ldots, 0<h \leq h_{0} .
$$

It is readily seen that $w_{h}^{n+1}$ satisfies

$$
\begin{cases}(\Delta-\lambda) w_{h}^{n+1}=F_{\lambda}\left(\cdot, u_{h}^{n}\right) & \text { on } \Omega \\ w_{h}^{n+1}=g & \text { on } \partial \Omega .\end{cases}
$$


Lemma 4.5. Let $N=2$ or 3 . Let $C^{0, \beta}(\bar{\Omega})$ be the Hölder space of continuous functions with exponent $\beta, 0<\beta<1$, on $\bar{\Omega}$. Then for $\varepsilon_{0}(N)$ satisfying (4.23), and for $\beta$ sufficiently small, we have a positive constant $K_{\varepsilon_{0}(N)}^{\prime}$ such that

$$
\begin{aligned}
\left\|u_{h}^{n+1}-w_{h}^{n+1}\right\|_{C^{0, \beta}(\bar{\Omega})} \leq K_{\varepsilon_{0}(N)}^{\prime} h^{\frac{1}{2}-\varepsilon_{0}(N)}\left[\left\|u_{\infty}\right\|_{H^{2}(\Omega)}+M_{\lambda} \alpha_{\lambda}^{n}\left\|e^{0}\right\|_{L^{2}(\Omega)}\right. \\
\left.+\left\|F_{\lambda}\left(\cdot, u_{h}^{n}\right)-F_{\lambda}\left(\cdot, u^{n}\right)\right\|_{L^{2}(\Omega)}\right],
\end{aligned}
$$

for $n=0,1,2, \ldots, 0<h \leq h_{0}$, where $K_{\varepsilon_{0}(3)}^{\prime}$ may grow unbounded as $\varepsilon_{0}(3) \downarrow 0$ when $N=3$.

Proof. We use the Sobolev imbedding theorem (cf. [6, Theorem 2.1.3], e.g.)

$$
\|f\|_{C^{0, \beta}(\bar{\Omega})} \leq C\|f\|_{H^{s}(\Omega)}, \quad \text { for } \quad 0<\beta=s-\frac{N}{2}<1,
$$

to get

$$
\left\|u_{h}^{n+1}-w_{h}^{n+1}\right\|_{C^{0, \beta}(\bar{\Omega})} \leq c_{\varepsilon_{0}}\left\|u_{h}^{n+1}-w_{h}^{n+1}\right\|_{H^{\frac{3}{2}+\varepsilon_{0}(N)}(\Omega)},
$$

where

$$
\left.\begin{array}{c}
0<\beta<\frac{1}{2} \\
\beta=\varepsilon_{0}(3)
\end{array}\right\} \quad \text { if } \quad\left\{\begin{array}{l}
N=2 \\
N=3 .
\end{array}\right.
$$

Note that $c_{\varepsilon_{0}} \uparrow \infty$ as $\varepsilon_{0}(3) \downarrow 0$. Continuing from the right-hand side of (4.31), we have

$$
\begin{aligned}
\left\|u_{h}^{n+1}-w_{h}^{n+1}\right\|_{C^{0, \beta}(\bar{\Omega})} & \leq c_{\varepsilon_{0}}\left\|\widetilde{S}_{\lambda}\left(\eta_{h}^{n+1}-\zeta_{h}^{n+1}\right)\right\|_{H^{\frac{3}{2}+\varepsilon_{0}(N)}(\Omega)} \\
& \left.\leq c_{\varepsilon_{0}} \cdot \widetilde{C}_{\varepsilon_{0}(N)}\left\|\eta_{h}^{n+1}-\zeta_{h}^{n+1}\right\|_{H^{\varepsilon_{0}(N)}} \quad \text { (by }(4.6)\right) .
\end{aligned}
$$

The rest of the proof follows from Lemma 4.3.

Lemma 4.6. Let $N=2$ or 3 . Then there exists a constant $K>0$ such that

$$
\begin{array}{r}
\left\|w_{h}^{n+1}-u_{n+1}\right\|_{C^{0, \beta}(\bar{\Omega})} \leq K\left\|F_{\lambda}\left(\cdot, u_{h}^{n}\right)-F_{\lambda}\left(\cdot, u_{n}\right)\right\|_{L^{2}(\Omega)} \\
\forall n=0,1,2, \ldots, 0<h \leq h_{0}, \text { where } 0<\beta<1 / 2 .
\end{array}
$$

Proof. From (3.45), (4.27), (4.30) and (4.32), we get

$$
\begin{aligned}
& \left\|w_{h}^{n+1}-u_{n+1}\right\|_{C^{0, \beta}(\bar{\Omega})} \leq c\left\|w_{h}^{n+1}-u_{n+1}\right\|_{H^{2}(\Omega)} \quad\left(\text { for } 0<\beta<\frac{1}{2}, \text { by }(4.30)\right) \\
& \leq c\left[\left\|\widetilde{S}_{\lambda}\left(\zeta_{h}^{n+1}-\mu_{n+1}\right)\right\|_{H^{2}(\Omega)}+\left\|\mathcal{V}_{\lambda}\left(F_{\lambda}\left(\cdot, u_{n}^{h}\right)-F_{\lambda}\left(\cdot, u_{n}\right)\right)\right\|_{H^{2}(\Omega)}\right] \\
& \leq c\left[\widetilde{C}_{\frac{1}{2}} \widetilde{\widetilde{C}}_{\frac{1}{2}}\left\|S_{\lambda}\left(\zeta_{h}^{n+1}-\mu_{n+1}\right)\right\|_{H^{\frac{3}{2}}(\partial \Omega)}\right. \\
& \left.\quad+\left\|\mathcal{V}_{\lambda}\left(F_{\lambda}\left(\cdot, u_{n}^{h}\right)-F_{\lambda}\left(\cdot, u_{n}\right)\right)\right\|_{H^{2}(\Omega)}\right] \quad(\text { by }(4.6),(4.7)) \\
& \leq c\left[\widetilde{C}_{\varepsilon_{0}(N)} \widetilde{\widetilde{C}}_{\varepsilon_{0}(N)}\left\|\mathcal{V}_{\lambda, \Gamma}\left(F_{\lambda}\left(\cdot, u_{h}^{n}\right)-F_{\lambda}\left(\cdot, u_{n}\right)\right)\right\|_{H^{\frac{3}{2}}(\partial \Omega)}\right. \\
& \left.\quad+\left\|\mathcal{V}_{\lambda}\left(F_{\lambda}\left(\cdot, u_{h}^{n}\right)-F_{\lambda}\left(\cdot, u_{n}\right)\right)\right\|_{H^{2}(\Omega)}\right] \\
& \leq c^{\prime}\left\|F_{\lambda}\left(\cdot, u_{h}^{n}\right)-F_{\lambda}\left(\cdot, u_{n}\right)\right\|_{L^{2}(\Omega)} \quad(\text { by }(4.2) \text { and }(4.4)) . \square
\end{aligned}
$$

Similar to Lemmas 4.5 and 4.6, we give the parallel estimates in the $L^{2}(\Omega)$ norms in Lemma 4.7. 
Lemma 4.7. We have

(4.34)

$$
\begin{gathered}
\left\|u_{h}^{n+1}-w_{h}^{n+1}\right\|_{L^{2}(\Omega)} \leq K h^{2}\left[\left\|u_{\infty}\right\|_{H^{2}(\Omega)}+M_{\lambda} \alpha_{\lambda}^{n}\left\|e^{0}\right\|_{L^{2}(\Omega)}\right. \\
\left.+\left\|F_{\lambda}\left(\cdot, u_{h}^{n}\right)-F_{\lambda}\left(\cdot, u_{n}\right)\right\|_{L^{2}(\Omega)}\right], \text { for some } K>0, \\
\left\|w_{h}^{n+1}-u_{n+1}\right\|_{L^{2}(\Omega)} \leq \frac{1}{\lambda+\lambda_{1}}\left\|F_{\lambda}\left(\cdot, u_{h}^{n}\right)-F_{\lambda}\left(\cdot, u_{n}\right)\right\|_{L^{2}(\Omega)}, \\
\forall n=0,1,2,3, \ldots, 0<h \leq h_{0} .
\end{gathered}
$$

Proof. From (4.12) and (4.27),

$$
\begin{aligned}
\left\|u_{h}^{n+1}-w_{h}^{n+1}\right\|_{L^{2}(\Omega)} & =\left\|\widetilde{S}_{\lambda}\left(\eta_{h}^{n+1}-\zeta_{h}^{n+1}\right)\right\|_{L^{2}(\Omega)} \\
& \left.\leq \widetilde{C}_{\frac{3}{2}}\left\|\eta_{h}^{n+1}-\zeta_{h}^{n+1}\right\|_{H^{-\frac{3}{2}}(\partial \Omega)} \quad \text { (by }(4.6)\right) .
\end{aligned}
$$

The rest follows from (4.32).

To show (4.35), we use that $u_{n+1}$ and $w_{h}^{n+1}$ satisfy, respectively, (3.43) and (4.28). Subtracting (4.28) from (3.43) and estimating as in (4.16) and (4.17), we obtain (4.35).

Corollary 4.8. (i) Let $N=2$ or 3 . Then for $\varepsilon_{0}(N)$ satisfying (4.23) and for $\beta>0$ sufficiently small, with $\beta$ being related to $\varepsilon_{0}(N)$ through (4.32), we have a constant $K_{\varepsilon_{0}(N)}^{\prime}>0$ such that

$$
\begin{aligned}
\left\|u_{h}^{n+1}-u_{n+1}\right\|_{C^{0, \beta}(\bar{\Omega})} \leq & K_{\varepsilon_{0}(N)}^{\prime} h^{\frac{1}{2}-\varepsilon_{0}(N)}\left[\left\|u_{\infty}\right\|_{H^{2}(\Omega)}+M_{\lambda} \alpha_{\lambda}^{n}\left\|e^{0}\right\|_{L^{2}(\Omega)}\right] \\
& +\left[\frac{1}{\lambda+\lambda_{1}}+K_{\varepsilon_{0}(N)}^{\prime} h^{\frac{1}{2}-\varepsilon_{0}(N)}\right]\left\|F_{\lambda}\left(\cdot, u_{h}^{n}\right)-F_{\lambda}\left(\cdot, u_{n}\right)\right\|_{L^{2}(\Omega)},
\end{aligned}
$$

$\forall n=0,1,2, \ldots, 0<h \leq h_{0}$, where $K_{\varepsilon_{0}(N)}^{\prime}$ may grow unbounded as $\varepsilon_{0}(3) \downarrow 0$.

(ii) There exists a constant $K>0$ such that

$$
\begin{gathered}
\left\|u_{h}^{n+1}-u_{n+1}\right\|_{L^{2}(\Omega)} \leq K h^{2}\left[\left\|u_{\infty}\right\|_{H^{2}(\Omega)}+M_{\lambda} \alpha_{\lambda}^{n}\left\|e^{0}\right\|_{L^{2}(\Omega)}\right]+\left(\frac{1}{\lambda+\lambda_{1}}+K h^{2}\right) \\
\cdot\left\|F_{\lambda}\left(\cdot, u_{h}^{n}\right)-F_{\lambda}\left(\cdot, u_{n}\right)\right\|_{L^{2}(\Omega)}, \\
\forall n=0,1,2, \ldots, 0<h \leq h_{0} .
\end{gathered}
$$

Proof. The inequality (4.36) follows from an application of the triangle inequality to (4.29) and (4.33), while (4.37) follows likewise from (4.34) and (4.35).

Theorem 4.9. Let $N=2$ or 3 and let $\varepsilon_{0}(N)$ be given by (4.23) and $\beta>0$ be sufficiently small, with $\beta$ being related to $\varepsilon_{0}(N)$ through (4.32). Then there exist $\bar{h}_{0}: 0<\bar{h}_{0} \leq h_{0}$ and $\rho_{\lambda}: 0<\rho_{\lambda}<1$, independent of $h$ and $n$, such that

$$
\begin{aligned}
& \left\|u_{h}^{n+1}-u_{n+1}\right\|_{C^{0, \beta}(\bar{\Omega})} \leq K_{\varepsilon_{0}(N)}^{\prime \prime} h^{\frac{1}{2}-\varepsilon_{0}(N)}\left[\left\|u_{\infty}\right\|_{H^{2}(\Omega)}+\rho_{\lambda}^{n+1}\left\|e^{0}\right\|_{L^{2}(\Omega)}\right] \\
& \left\|u_{h}^{n+1}-u_{n+1}\right\|_{L^{2}(\Omega)} \leq \bar{K} h^{2}\left[\left\|u_{\infty}\right\|_{H^{2}(\Omega)}+\rho_{\lambda}^{n+1}\left\|e^{0}\right\|_{L^{2}(\Omega)}\right]
\end{aligned}
$$


and

$$
a-\delta \leq u_{h}^{n+1}(x) \leq b+\delta, \quad \forall n=0,1,2, \ldots, 0<h \leq \bar{h}_{0},
$$

where $K_{\varepsilon_{0}(N)}^{\prime \prime}$ and $\bar{K}$ are constants independent of $h$ and $n$.

Proof. Since $0<\alpha_{\lambda}<1$, we can choose $\bar{h}_{0}>0$ so small that

$$
\begin{gathered}
{\left[K_{\varepsilon_{0}(N)}^{\prime} \bar{h}_{0}^{\frac{1}{2}-\varepsilon_{0}(N)}+\left(\alpha_{\lambda}+K_{\varepsilon_{0}(N)}^{\prime} M_{\lambda} \bar{h}_{0}^{\frac{1}{2}-\varepsilon_{0}(N)}\right)\left(\frac{K}{1-\rho_{\lambda}}\right) \bar{h}_{0}^{2}\right]} \\
\cdot\left[\left\|u_{\infty}\right\|_{H^{2}(\Omega)}+M_{\lambda}\left\|e^{0}\right\|_{L^{2}(\Omega)}\right] \leq \delta
\end{gathered}
$$

for some $\rho_{\lambda}$ : $0<\rho_{\lambda}<1$. By induction, we may assume

$$
u_{h}^{k}(x) \in[a-\delta, b+\delta], \forall x \in \bar{\Omega}, \quad k=0,1,2, \ldots, n, \quad 0<h \leq \bar{h}_{0} .
$$

Hence, similar to (4.18)-(4.20), we have (4.43)

$\left\|F\left(\cdot, u_{h}^{k}\right)-F\left(\cdot, u_{k}\right)\right\|_{L^{2}(\Omega)} \leq M_{\lambda}\left\|u_{h}^{k}-u_{k}\right\|_{L^{2}(\Omega)}, \quad k=0,1,2, \ldots, n, \quad 0<h \leq \bar{h}_{0}$.

Using (4.41) and (4.43) in (4.37), we obtain

$$
\begin{aligned}
\| u_{h}^{n+1} & -u_{n+1} \|_{L^{2}(\Omega)} \\
\leq & K h^{2}\left[\left\|u_{\infty}\right\|_{H^{2}(\Omega)}+M_{\lambda} \alpha_{\lambda}^{n}\left\|e^{0}\right\|_{L^{2}(\Omega)}\right] \\
& +\rho_{\lambda}\left\{K h^{2}\left[\left\|u_{\infty}\right\|_{H^{2}(\Omega)}+M_{\lambda} \alpha_{\lambda}^{n-1}\left\|e^{0}\right\|_{L^{2}(\Omega)}\right]+\rho_{\lambda}\left\|u_{h}^{n-1}-u_{n-1}\right\|_{L^{2}(\Omega)}\right\} \\
\leq & \cdots \\
\leq & K h^{2}\left(1+\rho_{\lambda}+\cdots+\rho_{\lambda}^{n}\right)\left\|u_{\infty}\right\|_{H^{2}(\Omega)} \\
& +K M_{\lambda} h^{2}\left(\alpha_{\lambda}^{n}+\rho_{\lambda} \alpha_{\lambda}^{n-1}+\cdots+\rho_{\lambda}^{n-1} \alpha_{\lambda}+\rho_{\lambda}^{n}\right)\left\|e^{0}\right\|_{L^{2}(\Omega)},
\end{aligned}
$$

where in the last inequality, we have used $u_{h}^{0}=u_{0}=\bar{u}$. Since $\alpha_{\lambda}<1$, we derive

$$
\begin{array}{r}
\left\|u_{h}^{n+1}-u_{n+1}\right\|_{L^{2}(\Omega)} \leq\left(\frac{K}{1-\rho_{\lambda}}\right) h^{2}\left[\left\|u_{\infty}\right\|_{H^{2}(\Omega)}+M_{\lambda}\left\|e^{0}\right\|_{L^{2}(\Omega)}\right] \\
\forall n=0,1,2, \ldots, \quad 0<h \leq \bar{h}_{0} .
\end{array}
$$

By applying (4.43) and (4.45) to (4.36), we then obtain

$$
\begin{array}{r}
\left\|u_{h}^{n+1}-u_{n+1}\right\|_{C^{0, \beta}(\bar{\Omega})} \leq \bar{K}_{\varepsilon_{0}(N)} h^{\frac{1}{2}-\varepsilon_{0}(N)}\left[\left\|u_{\infty}\right\|_{H^{2}(\Omega)}+M_{\lambda}\left\|e^{0}\right\|_{L^{2}(\Omega)}\right], \\
\forall n=0,1,2, \ldots, \quad 0<h \leq \bar{h}_{0},
\end{array}
$$


where

$$
\bar{K}_{\varepsilon_{0}(N)}=K_{\varepsilon_{0}(N)}^{\prime}+\left(\alpha_{\lambda}+K_{\varepsilon_{0}(N)}^{\prime} M_{\lambda} \bar{h}_{0}^{\frac{1}{2}-\varepsilon_{0}(N)}\right)\left(\frac{K}{1-\rho_{\lambda}}\right) \bar{h}_{0}^{\frac{3}{2}+\varepsilon_{0}(N)} .
$$

Thus, (4.40) is obtained from (4.42), (4.46), (4.47) and the induction hypothesis.

To get (4.39), we note that $\alpha_{\lambda}<\rho_{\lambda}$ from (4.41). Then, instead of (4.45), we obtain from (4.44)

$$
\begin{aligned}
\left\|u_{h}^{n+1}-u_{n+1}\right\|_{L^{2}(\Omega)} & \leq\left(\frac{K}{1-\rho_{\lambda}}\right) h^{2}\left\|u_{\infty}\right\|_{H^{2}(\Omega)}+K M_{\lambda}\left(\frac{\rho_{\lambda}^{n+1}}{\rho_{\lambda}-\alpha_{\lambda}}\right) h^{2}\left\|e^{0}\right\|_{L^{2}(\Omega)}, \\
& \leq\left(\frac{K}{1-\rho_{\lambda}}\right) h^{2}\left\|u_{\infty}\right\|_{H^{2}(\Omega)}+\left(\frac{1}{\bar{h}_{0}^{2}}\right) \rho_{\lambda}^{n+1} h^{2}\left\|e^{0}\right\|_{L^{2}(\Omega)} \\
& \forall n=0,1,2, \ldots, \quad 0<h \leq \bar{h}_{0},
\end{aligned}
$$

where we used (4.41). Thus, (4.39) follows from (4.48) by setting $\bar{K}=$ $\max \left\{\frac{K}{1-\rho_{\lambda}}, \frac{1}{h_{0}^{2}}\right\}$.

Finally, by using (4.40), (4.41) and then (4.48), we obtain (4.38) from (4.36). The proof is complete.

The inequality (4.40) is important because it guarantees that each numerical iterate $u_{h}^{n}(x)$ does not fall out of the range of validity, $\Omega \times[a-\delta, b+\delta]$, of the monotone iteration, and for the applicability of (4.18)-(4.20). It also partially explains why we need an assumption like $[\mathrm{H}]$.

Theorem 4.10. Let $\left\{\mathcal{S}_{h} \mid 0<h \leq h_{0}\right\}$ be a family of $(\ell, m)$-systems on $\partial \Omega$ with $\ell \geq 2$ and $m \geq 1$, and let $N=2$ or 3 . Assume $[H]$, so that there exists a small number $\delta>0$ for which (4.13) holds. Then there exist constants $K_{1}>0, K_{2}>0$, and an $\bar{h}_{0}: 0<\bar{h}_{0} \leq h_{0}$, such that for any $r: 0 \leq r \leq 2$, we have

$$
\left\|u_{h}^{n}-u_{\infty}\right\|_{H^{r}(\Omega)} \leq K_{1} h^{2-r}\left\|u_{\infty}\right\|_{H^{2}(\Omega)}+K_{2} \rho_{\lambda}^{n}\left\|e^{0}\right\|_{L^{2}(\Omega)}
$$

and

$$
a-\delta \leq u_{h}^{n}(x) \leq b+\delta,
$$

for all $n=0,1,2, \ldots, h$ : $0<h \leq \bar{h}_{0}$, where $e^{0}=u_{\infty}-\bar{u}$ and $0<\rho_{\lambda}<1$. Therefore, $u_{h}^{n}$ converges to $u_{\infty}$ as $n$ tends to $\infty$ and $h$ tends to 0 .

Proof. Similar to the procedures in (4.26), by using (4.39) and (4.43), we have

$$
\begin{aligned}
\left\|\eta_{h}^{n+1}-\zeta_{h}^{n+1}\right\|_{H^{s}(\partial \Omega)} \leq & \widetilde{C}_{\frac{1}{2}, s} h^{\frac{1}{2}-s}\left\|\zeta_{h}^{n+1}\right\|_{H^{\frac{1}{2}}(\partial \Omega)} \\
\leq\left(\widetilde{C}_{\frac{1}{2}, s} \widetilde{C}_{\frac{1}{2}}\right) h^{\frac{1}{2}-s}\left[C\left\|u_{\infty}\right\|_{H^{2}(\Omega)}+\overline{\bar{C}}_{0} M_{\lambda} \alpha_{\lambda}^{n}\left\|e^{0}\right\|_{L^{2}(\Omega)}\right. & \left.\quad \overline{\bar{C}}_{0}\left\|F_{\lambda}\left(\cdot, u_{h}^{n}\right)-F_{\lambda}\left(\cdot, u_{n}\right)\right\|_{L^{2}(\Omega)}\right] \\
\leq & \left(\widetilde{C}_{\frac{1}{2}, s} \widetilde{C}_{\frac{1}{2}}\right) h^{\frac{1}{2}-s}\left[C\left\|u_{\infty}\right\|_{H^{2}(\Omega)}+\overline{\bar{C}}_{0} M_{\lambda} \alpha_{\lambda}^{n}\left\|e^{0}\right\|_{L^{2}(\Omega)}\right. \\
& +\overline{\bar{C}}_{0} M_{\lambda} \bar{K} h^{2}\left(\left\|u_{\infty}\right\|_{H^{2}(\Omega)}+\rho_{\lambda}^{n}\left\|e^{0}\right\|_{L^{2}(\Omega)}\right] \\
\leq & C_{1} h^{\frac{1}{2}-s}\left[\left\|u_{\infty}\right\|_{H^{2}(\Omega)}+\rho_{\lambda}^{n}\left\|e^{0}\right\|_{L^{2}(\Omega)}\right]
\end{aligned}
$$


where $-(\ell+1) \leq s \leq 1 / 2$. From here we then have, cf. the proof of Lemma 4.7,

$$
\begin{aligned}
\left\|u_{h}^{n+1}-w_{h}^{n+1}\right\|_{H^{s+\frac{3}{2}}(\Omega)} & =\left\|\widetilde{S}_{\lambda}\left(\eta_{h}^{n+1}-\zeta_{h}^{n+1}\right)\right\|_{H^{s+\frac{3}{2}}(\Omega)} \leq \widetilde{C}_{s}\left\|\eta_{h}^{n+1}-\zeta_{h}^{n+1}\right\|_{H^{s}(\partial \Omega)} \\
& \leq C_{2} h^{\frac{1}{2}-s}\left[\left\|u_{\infty}\right\|_{H^{2}(\Omega)}+\rho_{\lambda}^{n}\left\|e^{0}\right\|_{L^{2}(\Omega)}\right] \quad(\text { by }(4.50)) .
\end{aligned}
$$

Let $r=s+\frac{3}{2}$ in (4.51). Then

$$
\left\|u_{h}^{n+1}-w_{h}^{n+1}\right\|_{H^{r}(\Omega)} \leq C_{2} h^{2-r}\left[\left\|u_{\infty}\right\|_{H^{2}(\Omega)}+\rho_{\lambda}^{n}\left\|e^{0}\right\|_{L^{2}(\Omega)}\right],
$$

where $-\ell+\frac{1}{2} \leq r \leq 2$.

For $0 \leq r \leq 2$, we have

$$
\begin{aligned}
\left\|w_{h}^{n+1}-u_{n+1}\right\|_{H^{r}(\Omega)} & \leq\left\|w_{h}^{n+1}-u_{n+1}\right\|_{H^{2}(\Omega)} \\
& \left.\leq \bar{C}_{0}\left\|F_{\lambda}\left(\cdot, u_{h}^{n}\right)-F_{\lambda}\left(\cdot, u_{n}\right)\right\|_{L^{2}(\Omega)} \quad \text { (by }(2.13) \text { and }(4.28)\right) \\
& \leq \bar{C}_{0} M_{\lambda}\left\|u_{h}^{n}-u_{n}\right\|_{L^{2}(\Omega)} \quad(\text { by }(4.43)) \\
& \leq\left(\bar{C}_{0} M_{\lambda} K\right) h^{2}\left[\left\|u_{\infty}\right\|_{H^{2}(\Omega)}+\rho_{\lambda}^{n}\left\|e^{0}\right\|_{L^{2}(\Omega)}\right] \quad(\text { by }(4.39)) .
\end{aligned}
$$

Combining (4.52) and (4.53), we obtain

$$
\begin{array}{r}
\left\|u_{h}^{n+1}-u_{n+1}\right\|_{H^{r}(\Omega)} \leq C_{3} h^{2-r}\left[\left\|u_{\infty}\right\|_{H^{2}(\Omega)}+\rho_{\lambda}^{n}\left\|e^{0}\right\|_{L^{2}(\Omega)}\right], \quad 0 \leq r \leq 2, \\
\forall n=0,1,2, \ldots, 0<h \leq \bar{h}_{0} .
\end{array}
$$

Also,

$$
\begin{aligned}
\left\|u_{n+1}-u_{\infty}\right\|_{H^{r}(\Omega)} & \leq\left\|u_{n+1}-u_{\infty}\right\|_{H^{2}(\Omega)} \\
& \left.\leq C_{\lambda}\left\|F_{\lambda}\left(\cdot, u_{h}\right)-F_{\lambda}\left(\cdot, u_{\infty}\right)\right\|_{L^{2}(\Omega)} \quad \text { (by }(4.16)\right) \\
& \leq C_{\lambda} M_{\lambda}\left\|u_{n}-u_{\infty}\right\|_{L^{2}(\Omega)} \\
& \leq C_{\lambda} M_{\lambda} \alpha_{\lambda}^{n}\left\|e^{0}\right\|_{L^{2}(\Omega)} \quad(\text { by }(4.14)) .
\end{aligned}
$$

Then, upon noting $\alpha_{\lambda}<\rho_{\lambda}$, we see that (4.49) follows from (4.54) and (4.55).

Let $\mu_{\infty}$ be the limit of the simple-layer densities $\left\{\mu_{n}\right\}_{1}^{\infty}$ given in Corollary 3.3. We give the error estimate $\left\|\eta_{h}^{n}-\mu_{\infty}\right\|$ in the following theorem.

Theorem 4.11. Let $N=2$ or 3. Let $\left\{\mathcal{S}_{h} \mid 0<h \leq h_{0}\right\}$ be a family of $(\ell, m)$ systems with $\ell \geq 2$ and $m \geq 1$. Then for $s:-\frac{3}{2} \leq s \leq \frac{1}{2}$, there are constants $K_{1}>0$ and $K_{2}>0$ such that

$$
\begin{array}{r}
\left\|\eta_{h}^{n}-\mu_{\infty}\right\|_{H^{s}(\partial \Omega)} \leq K_{1} h^{\frac{1}{2}-s}\left\|u_{\infty}\right\|_{H^{2}(\Omega)}+K_{2} \rho_{\lambda}^{n}\left\|e^{0}\right\|_{L^{2}(\Omega)}, \\
\forall n=0,1,2, \ldots, \quad h: 0<h \leq \bar{h}_{0} .
\end{array}
$$


Proof. First, from (4.21) and (3.47), we have that for $s \leq 1 / 2$,

$$
\begin{aligned}
&\left\|\zeta_{h}^{n+1}-\mu_{n}\right\|_{H^{s}(\partial \Omega)}=\left\|S_{\lambda}^{-1}\left[\mathcal{V}_{\lambda, \Gamma}\left(F_{\lambda}\left(\cdot, u_{h}^{n}\right)-F_{\lambda}\left(\cdot, u_{n}\right)\right)\right]\right\|_{H^{s}(\partial \Omega)} \\
& \leq \widetilde{\widetilde{C}}_{s+1}\left\|\mathcal{V}_{\lambda, \Gamma}\left[F_{\lambda}\left(\cdot, u_{h}^{n}\right)-F_{\lambda}\left(\cdot, u_{n}\right)\right]\right\|_{H^{\frac{3}{2}}(\partial \Omega)} \\
& \quad(\text { for any } s \in \mathbb{R}, \text { by }(4.7)) \\
&\left.\leq K\left\|u_{h}^{n}-u_{n}\right\|_{L^{2}(\Omega)} \quad \quad \quad \text { by }(4.2) \text { and }(4.43)\right) \\
& \leq K^{\prime} h^{2}\left[\left\|u_{\infty}\right\|_{H^{2}(\Omega)}+\rho_{\lambda}^{n}\left\|e^{0}\right\|_{L^{2}(\Omega)}\right], \text { from }(4.39) .
\end{aligned}
$$

Also, from (4.50),

$$
\left\|\eta_{h}^{n+1}-\zeta_{h}^{n+1}\right\|_{H^{s}(\partial \Omega)} \leq K^{\prime \prime} h^{\frac{1}{2}-s}\left[\left\|u_{\infty}\right\|_{H^{2}(\Omega)}+\rho_{\lambda}^{n}\left\|e^{0}\right\|_{L^{2}(\Omega)}\right], \quad-(\ell+1) \leq s \leq \frac{1}{2} .
$$

Similarly, from (3.47), (3.48), and the proofs of (4.57) and Lemma 4.1, we have

$$
\left\|\mu_{n}-\mu_{\infty}\right\|_{H^{s}(\partial \Omega)} \leq K^{\prime \prime \prime} \alpha_{\lambda}^{n}\left\|e^{0}\right\|_{L^{2}(\Omega)} \quad \text { for } \quad s \leq \frac{1}{2} .
$$

From (4.57)-(4.59) and the triangle inequality, (4.56) follows.

In our proofs above, for the $S_{h}^{\ell, m}(\partial \Omega)$-systems we have used, because of the need of the inverse inequality we require that $|\alpha| \leq m$ (cf. the proof of Lemma 4.2 for $\alpha), \alpha=-\frac{1}{2}$, leading to $m \geq 1$. Thus piecewise constant boundary element spaces $S_{h}^{1,0}(\partial \Omega)$ are excluded. However, this restriction may be relaxed for $N=2$ if we use $S_{h}^{d}$, the space of smoothest splines of degree $d$ with respect to a quasi-uniform mesh on $\partial \Omega$, which is a smooth Jordan curve in $\mathbb{R}^{2}$. In this case the approximation property (4.8) holds for all $s \leq t \leq d+1, s<d+\frac{1}{2}$, and the inverse property (4.9) holds for $s \leq t<d+\frac{1}{2}$ [12]. By [14, Corollary 4], in (4.24) we now have

$$
\begin{gathered}
\left\|\eta_{h}^{n+1}-\zeta_{h}^{n+1}\right\|_{H^{s}(\partial \Omega)} \leq C h^{t-s}\left\|\zeta_{h}^{n+1}\right\|_{H^{t}(\partial \Omega)}-(d+2) \leq s \leq t \leq d+1, \\
s<d+\frac{1}{2}, \quad t>-\left(d+\frac{3}{2}\right), \quad d \geq 0 .
\end{gathered}
$$

Therefore, all of the lemmas and theorems after Lemma 4.2 in this section remain valid for these $S_{h}^{d}$ systems with a quasiuniform mesh on $\partial \Omega$ when $N=2$. In particular, we state the following.

Theorem 4.12. Let $N=2$ and let $\left\{\mathcal{S}_{h} \mid 0<h \leq h_{0}\right\}$ be a family of $S_{h}^{d}$ spaces of smoothest splines of degree $d\left(\in \mathbb{Z}^{+}\right)$with respect to a quasiuniform mesh on $\partial \Omega$. Under otherwise the same assumptions as in Theorems 4.10 and 4.11, we have $K_{i}>0, i=1,2,3,4$, and $\bar{h}_{0}: 0<\bar{h}_{0} \leq h_{0}, 0<\rho_{\lambda}<1$, such that

$$
\begin{aligned}
\left\|u_{h}^{n}-u_{\infty}\right\|_{H^{2}(\Omega)} & \leq K_{1} h^{2-r}\left\|u_{\infty}\right\|_{H^{2}(\Omega)}+K_{2} \rho_{\lambda}^{n}\left\|e^{0}\right\|_{L^{2}(\Omega)}, \\
\left\|\eta_{h}^{n}-u_{\infty}\right\|_{H^{s}(\partial \Omega)} & \leq K_{3} h^{\frac{1}{2}-s}\left\|u_{\infty}\right\|_{H^{2}(\Omega)}+K_{4} \rho_{\lambda}^{n}\left\|e^{0}\right\|_{L^{2}(\Omega)}
\end{aligned}
$$

$\left(K_{3}\right.$ and $K_{4}$ depend on $\left.s\right)$,

$\forall r: 0 \leq r \leq 2, \quad n=0,1,2, \ldots, \quad h: 0<h \leq \bar{h}_{0} ; \quad s:-(d+2) \leq s<1 / 2$. 
Remark 4.13. The error estimates obtained in Theorems $4.10-4.12$ are all of the separable form

$$
K_{1} h^{\gamma}\left\|u_{\infty}\right\|_{H^{2}(\Omega)}+K_{2} \rho_{\lambda}^{n}\left\|e^{0}\right\|_{L^{2}(\Omega)}, \quad \gamma>0 .
$$

It is obvious that these estimates are asymptotically tight.

Remark 4.14. Error estimates of $\left\|u_{h}^{n}-u_{\infty}\right\|_{H^{r}(\Omega)}$ with respect to higher-order Sobolev space norms $H^{r}(\Omega)$, with $r>2$, and for space dimension $N>3$, are possible if we make an assumption like

$\left\|F_{\lambda}\left(\cdot, u_{1}(\cdot)\right)-F_{\lambda}\left(\cdot, u_{2}(\cdot)\right)\right\|_{H^{m}(\Omega)} \leq \widetilde{M}_{\lambda}\left\|u_{1}-u_{2}\right\|_{H^{m}(\Omega)}, \quad m \geq 1, \forall u_{1}, u_{2} \in H^{m}(\Omega)$,

for some sufficiently small $\widetilde{M}_{\lambda}$, to supersede (4.20). But (4.61) appears unnatural, and leads to cumbersome notation. So we do not go into this.

\section{Higher than OPtimal-ORDER ERROR ESTIMATES FOR NONLINEARITIES THAT ARE SEPARABLE}

A special feature of boundary elements for nonlinear PDEs is that for an important class of nonlinearities, one can obtain higher than "optimal order" error estimates with respect to the $h$-parameter. Let us assume that $F(x, u)$ in $(1.1)$ is separable, of the form

$$
F(x, u)=a_{0}(x) f(u)+f_{0}(x),
$$

where $a_{0}, f_{0} \in C^{\infty}(\bar{\Omega})$, and $f \in C^{\infty}(\mathbb{R})$, and that $g(x) \equiv 0$ on $\partial \Omega$.

Lemma 5.1. Let $\left\{\mathcal{S}_{h} \mid 0<h \leq h_{0}\right\}$ be $S_{h}^{\ell, m}(\partial \Omega)$-systems with $\ell \geq 2$ and $m \geq 1$ when $N=2$ or 3 , or be $S_{h}^{d}$-systems of smoothest splines of degree $d$ with quasiuniform mesh when $N=2$. Then there are $\bar{h}_{0}: 0<\bar{h}_{0} \leq h_{0}, \rho_{\lambda}: 0<\rho_{\lambda}<1$, and constants $K_{1}>0, K_{2}>0$ such that

$\left\|\eta_{h}^{n+1}-\zeta_{h}^{n+1}\right\|_{H^{-3 / 2}(\partial \Omega)} \leq K_{1} h^{2+\sigma}\left[\|u\|_{H^{2}(\Omega)}+\rho_{\lambda}^{n}\left\|e^{0}\right\|_{L^{2}(\Omega)}+K_{2}\right], \quad \forall \sigma: 0<\sigma<1$,

for all $0<h \leq \bar{h}_{0}, n=0,1,2, \ldots$, provided that $F(x, u)$ is of the form (5.1), and $g \equiv 0$. In particular, $K_{2}=0$ if $a_{0}(x)$ and $f_{0}(x)$ in (5.1) are constant functions.

Proof. We first recall the Sloboditskii norm $\|u\|_{\sigma}^{2} \equiv|u|_{\sigma}^{2}+\|u\|_{L^{2}(\Omega)}^{2}$, where

$$
|u|_{\sigma}^{2}=\iint_{\Omega \times \Omega} \frac{|u(x)-u(y)|^{2}}{|x-y|^{N+2 \sigma}} d x d y
$$

for $0<\sigma<1$. It is known that the Sloboditskii norm is equivalent to the Sobolev space norm $\|\cdot\|_{H^{\sigma}(\Omega)}^{2}$. From (5.1), we have, for $a-\delta \leq u(x) \leq b+\delta, x \in \Omega$,

$$
\begin{aligned}
\left|F_{\lambda}(x, u(x))-F_{\lambda}(y, u(y))\right| & \\
=\mid \lambda[u(x)-u(y)]-\left[a_{0}(x)-a_{0}(y)\right] f(u(x))-a_{0}(y)[ & f(u(x))-f(u(y))] \\
+ & +\left[f_{0}(x)-f_{0}(y)\right] \mid
\end{aligned}
$$


from which it follows that

$$
\begin{aligned}
\left|F_{\lambda}(\cdot, u)\right|_{\sigma} & \leq \lambda|u|_{\sigma}+\|f\|_{C{ }^{[a-\delta, b+\delta]}}\left|a_{0}\right|_{\sigma}+\left\|a_{0}\right\|_{C^{0}(\bar{\Omega})}\left\|f^{\prime}\right\|_{C^{0}[a-\delta, b+\delta]}|u|_{\sigma}+\left|f_{0}\right|_{\sigma} \\
& \equiv C_{1}|u|_{\sigma}+C_{2}, \quad 0<\sigma<1 .
\end{aligned}
$$

Because of (4.48), we can get

$$
\left\|F_{\lambda}\left(\cdot, u_{h}^{n}\right)-F_{\lambda}\left(\cdot, u_{\infty}\right)\right\|_{L^{2}(\Omega)} \leq M_{\lambda}\left\|u_{h}^{n}-u_{\infty}\right\|_{L^{2}(\Omega)} .
$$

Similarly as in the proof of Lemma 4.5 , we obtain

$$
\begin{aligned}
& \left\|\eta_{h}^{n+1}-\zeta_{h}^{n+1}\right\|_{H^{-3 / 2}(\partial \Omega)} \leq C_{3} h^{2+\sigma}\left\|\zeta_{h}^{n+1}\right\|_{H^{\frac{1}{2}+\sigma}(\partial \Omega)} \\
& \left.\quad \leq C_{4} h^{2+\sigma}\left\|F_{\lambda}\left(\cdot, u_{h}^{n}\right)\right\|_{H^{\sigma}(\Omega)} \quad \text { (because } g=0 \text { in }(4.21)\right) \\
& \quad \leq C_{4} h^{2+\sigma}\left[\left|F_{\lambda}\left(\cdot, u_{h}^{n}\right)\right|_{\sigma}+\left\|F_{\lambda}\left(\cdot, u_{h}^{n}\right)-F_{\lambda}\left(\cdot, u_{\infty}\right)\right\|_{L^{2}(\Omega)}+\left\|F_{\lambda}\left(\cdot, u_{\infty}\right)\right\|_{L^{2}(\Omega)}\right] \\
& \quad \leq C_{4} h^{2+\sigma}\left[C_{1}\left|u_{h}^{n}\right|_{\sigma}+C_{2}+M_{\lambda}\left\|u_{h}^{n}-u_{\infty}\right\|_{L^{2}(\Omega)}+C_{5}\left\|u_{\infty}\right\|_{H^{2}(\Omega)}\right] \\
& \quad \leq C_{4} h^{2+\sigma}\left[C_{6}\left(\left\|u_{h}^{n}-u_{\infty}\right\|_{H^{\sigma}(\Omega)}+C_{7}\left\|u_{\infty}\right\|_{H^{2}(\Omega)}\right)+C_{2}\right] \quad \text { by (5.3) and (5.4)) } \\
& \quad \leq C_{8} h^{2+\sigma}\left[\left\|u_{\infty}\right\|_{H^{2}(\Omega)}+\rho_{\lambda}^{n}\left\|e^{0}\right\|_{L^{2}(\Omega)}+C_{9}\right] \quad \text { (by Theorem 4.10). } \square
\end{aligned}
$$

Now, the following is obvious.

Theorem 5.2. Assume the same conditions as in Lemma 5.1. Then there are three constants $C_{i}>0, i=1,2,3$, such that

$$
\begin{aligned}
\left\|u_{h}^{n}-u_{\infty}\right\|_{H^{r}(\Omega)} \leq & C_{1} \rho_{\lambda}^{n}\left\|e^{0}\right\|_{L^{2}(\Omega)}+C_{2} h^{2-r+\sigma}\left(\left\|u_{\infty}\right\|_{H^{2}(\Omega)}+C_{3}\right) \\
& \forall h: 0<h \leq \bar{h}_{0}, \quad n=0,1,2, \ldots, \quad 0 \leq \sigma<1, \quad 0 \leq r \leq 2 .
\end{aligned}
$$

In particular, $C_{3}=0$ if $a_{0}(x)$ is a constant function and $f_{0}(x) \equiv 0$ in (5.1).

\section{Neumann And Robin Boundary CONDITIONS}

The treatments in $\S 4$ and $\S 5$ deal with the Dirichlet boundary condition. We now consider the case when the boundary condition is

$$
\frac{\partial}{\partial n} u(x)+\alpha(x) u(x)=g(x), \quad x \in \partial \Omega,
$$

where $\alpha(x) \geq 0, \alpha \in C^{\infty}(\partial \Omega)$. The condition (6.1) corresponds to a Neumann or a Robin boundary value problem depending on whether $\alpha(x)$ is or is not identically equal to zero.

For (6.1), we formulate a corresponding assumption similar to [H]. Let $\lambda_{1}^{\prime}$ be the smallest eigenvalue of the operator $\left(-\Delta,\left.\left[\frac{\partial}{\partial n}+\alpha(\cdot)\right]\right|_{\Gamma}\right)$. Then $\lambda_{1}^{\prime}=0$ if $\alpha(x) \equiv 0$ and $\lambda_{1}^{\prime}>0$ if $\alpha(x) \geq 0$ but $\alpha(x) \not \equiv 0$. We assume:

$[\mathrm{H}]^{\prime}$ There exist constants $\gamma_{1}^{\prime}$ and $\gamma_{2}^{\prime}$ such that

$$
-\lambda_{1}^{\prime}<\gamma_{1}^{\prime} \leq \frac{\partial F(x, u)}{\partial u} \leq \gamma_{2}^{\prime}, \quad \forall(x, u) \in \bar{Q} \equiv \bar{\Omega} \times[a, b] .
$$


Similar to (4.13), we now have that for a sufficiently small $\delta>0$, there exist $\tilde{\gamma}_{1}^{\prime}$ and $\tilde{\gamma}_{2}^{\prime}$ such that

$$
-\lambda_{1}^{\prime}<\tilde{\gamma}_{1}^{\prime} \leq \frac{\partial F(x, u)}{\partial u} \leq \tilde{\gamma}_{2}^{\prime}, \quad \forall(x, u) \in \bar{Q}_{\delta} \equiv \bar{\Omega} \times[a-\delta, b+\delta] .
$$

Let $\lambda>0$ be chosen sufficiently large such that

$$
M_{\lambda}^{\prime} \equiv \sup _{(x, u) \in \bar{Q}_{\delta}}\left[\lambda-\frac{\partial F(x, u)}{\partial u}\right]>0 .
$$

Then

$$
0<M_{\lambda}^{\prime}<\lambda-\tilde{\gamma}_{1}^{\prime}
$$

Lemma 6.1. Let the assumptions in Theorem 2.2 and (6.1)-(6.4) hold. Let $u_{n+1}$ be iterated according to

$$
\begin{cases}(\Delta-\lambda) u_{n+1}(x)=F_{\lambda}\left(x, u_{n}(x)\right), & x \in \Omega, \\ \frac{\partial}{\partial n} u_{n+1}(x)+\alpha(x) u_{n+1}(x)=g(x), & x \in \partial \Omega, g \in C^{\infty}(\partial \Omega),\end{cases}
$$

and let $u_{\infty}$ be the limit as in (2.16). Denote $e^{n}=u_{\infty}-u_{n}$. Then we have

$$
\left\|e^{n+1}\right\|_{L^{2}(\Omega)} \leq \alpha_{\lambda}^{\prime n+1}\left\|e^{0}\right\|_{L^{2}(\Omega)}, \quad n=0,1,2, \ldots,
$$

where

$$
0<\alpha_{\lambda}^{\prime} \equiv \frac{\lambda-\tilde{\gamma}_{1}^{\prime}}{\lambda+\lambda_{1}^{\prime}}<1
$$

Proof. Refer to the proof of Lemma 4.1. Instead of (4.16), we now have

$$
\begin{cases}(\Delta-\lambda) e^{n+1}(x)=F_{\lambda}\left(x, u_{\infty}(x)\right)-F_{\lambda}\left(x, u_{n}(x)\right), & x \in \Omega, \\ \frac{\partial}{\partial n} e^{n+1}(x)+\alpha(x) e^{n+1}(x)=0, & x \in \partial \Omega .\end{cases}
$$

Multiplying the above by $-e^{n+1}$ and integrating by parts, we get

$$
\begin{array}{r}
\int_{\partial \Omega} \alpha\left|e^{n+1}\right|^{2} d \sigma+\int_{\Omega}\left|\nabla e^{n+1}\right|^{2} d x+\lambda \int_{\Omega}\left|e^{n+1}\right|^{2} d x \\
\leq\left\|F_{\lambda}\left(\cdot, u_{\infty}\right)-F_{\lambda}\left(\cdot, u_{n}\right)\right\|_{L^{2}(\Omega)}\left\|e^{n+1}\right\|_{L^{2}(\Omega)} .
\end{array}
$$

But

$$
\inf _{v \in H^{1}(\Omega)} \frac{\left[\int_{\partial \Omega} \alpha|v|^{2} d \sigma+\int_{\Omega}|\nabla v|^{2} d x\right]}{\|v\|_{L^{2}(\Omega)}^{2}}=\lambda_{1}^{\prime} .
$$

Thus,

$$
\left(\lambda+\lambda_{1}^{\prime}\right)\left\|e^{n+1}\right\|_{L^{2}(\Omega)}^{2} \leq\left\|F_{\lambda}\left(\cdot, u_{\infty}\right)-F_{\lambda}\left(\cdot, u_{n}\right)\right\|_{L^{2}(\Omega)}\left\|e^{n+1}\right\|_{L^{2}(\Omega)} .
$$

The rest of the arguments are the same as in the proof of Lemma 4.1. 
Proceeding as in $\S 4$, we now note where the places are that need to be adjusted to accommodate the boundary condition (6.1). The iterate $u_{n+1}(\cdot)$ satisfying $(6.5)$ is still represented as in (3.8), but $\eta_{n+1}$ is determined from the BIE

$$
\begin{aligned}
\mathcal{B}_{\lambda} u_{n+1}(x) & \equiv \frac{1}{2} \eta_{n+1}(x)+\int_{\partial \Omega}\left[\frac{\partial}{\partial n_{x}} E(x-y ; \lambda)+\alpha(x) E(x-y ; \lambda)\right] \eta_{n+1}(y) d \sigma_{y} \\
& =g(x)+\alpha(x)\left(\mathcal{V}_{\lambda, \Gamma} F\left(\cdot, u_{n}\right)\right)(x)+\frac{\partial}{\partial n}\left(\mathcal{V}_{\lambda} F\left(\cdot, u_{n}\right)\right)(x), \quad x \in \partial \Omega,
\end{aligned}
$$

instead of (3.47). Note that $\mathcal{B}_{\lambda}$ is a strongly elliptic pseudodifferential operator of order $0\left(=2 \alpha\right.$ according to the notation in [10]) with principal symbol $\frac{1}{2}$ such that

$$
\mathcal{B}_{\lambda}: H^{s}(\partial \Omega) \longrightarrow H^{s}(\partial \Omega) \text { isomorphically, } \forall s \in \mathbb{R} \text {. }
$$

Therefore, (6.6) is uniquely solvable.

The Galerkin boundary element method for (6.5) is almost the same as in (4.10)(4.12), except that (4.11) is replaced by

(ii) $^{\prime}$ For each $n=0,1,2, \ldots$, and $h: 0<h \leq h_{0}$, find $\eta_{h}^{n+1} \in \mathcal{S}_{h}$ such that

$$
\left\langle\mathcal{B}_{\lambda} \eta_{h}^{n+1}, \phi_{h}\right\rangle_{L^{2}(\partial \Omega)}=\left\langle *, \phi_{h}\right\rangle_{L^{2}(\partial \Omega)}, \quad \forall \phi_{h} \in \mathcal{S}_{h}
$$

where $*$ denotes the sum in the last equality of (6.6), but with $u_{h}^{n}$ taking the place of $u_{h}$.

Next, we let $\zeta_{h}^{n+1}$ be the unique solutions of the BIE

$$
\left(\mathcal{B}_{\lambda} \zeta_{h}^{n+1}\right)(x)=g(x)+\alpha(x)\left(\mathcal{V}_{\lambda, \Gamma} F\left(\cdot, u_{h}^{n}\right)\right)(x)+\frac{\partial}{\partial n}\left(\mathcal{V}_{\lambda} F\left(\cdot, u_{h}^{n}\right)\right)(x), \quad x \in \partial \Omega
$$

(instead of (4.21))

from which we define $w_{h}^{n+1}(x)$ just as in (4.27).

It is now routine to check that beginning from (4.21) in Lemma 4.2 and ending at (4.60), all the estimates remain valid. Indeed, we obtain the same results under a less restrictive assumption on $S_{h}^{\ell, m}(\partial \Omega)$ - here we only require that $\ell \geq 1$ (and $m \geq 0$ ) because $2 \alpha$, the order of $\mathcal{B}_{\lambda}$, is zero. Thus the requirement that $|\alpha| \leq m$ made in [10, Corollary 2.1] and [14, Corollary 3] is now automatically satisfied with $m \geq 0$.

\section{Numerical examples}

The theory developed so far was molded by computations of concrete examples of semilinear PDEs. In the following, we will present several such examples featuring various situations, with numerical solutions illustrated by computer graphics.

The steps that we take are indicated by a flowchart next. For definiteness, we only consider the Dirichlet boundary condition for $\Omega \equiv D_{1}$, the unit open disk in 


\section{FLOW-CHART}

1. Initialize: Take an initial guess $w_{0}(x), x \in \Omega$.

Set $j=0$. Select $\lambda>0$ to satisfy (2.9).

2. Compute the volume potential

$$
V_{\lambda, j}(x) \equiv \int_{\Omega} E(x-y)\left[\lambda w_{j}(y)-F\left(y, w_{j}(y)\right)\right] d y, \quad x \in \bar{\Omega} .
$$

3. Solve the unknown simple-layer density $\eta_{j+1}(\cdot)$ from the boundary integral equation

$\int_{\partial \Omega} E(x-y) \eta_{j+1}(y) d \sigma_{y}=g(x)-\int_{\Omega} E(x-y)\left[\lambda w_{j}(y)-F\left(y, w_{j}(y)\right)\right] d y$,
$x \in \partial \Omega$.

4. Compute the solution

$w_{j+1}(x)=\int_{\partial \Omega} E(x-y) \eta_{j+1}(y) d \sigma_{y}+\int_{\Omega} E(x-y)\left[\lambda w_{j}(y)-F\left(y, w_{j}(y)\right)\right] d y$, $x \in \bar{\Omega}$.

5. If $\left\|w_{j+1}-w_{j}\right\|_{L^{2}(\Omega)}<\varepsilon$, exit. Output and stop.

6. Else $j:=j+1$;

continue.

$\mathbb{R}^{2}$. The boundary value problem is of the form

$$
\begin{cases}\Delta u(x)-F(x, u(x))=0, & x \in D_{1} \\ u(x)=g(x), & x \in \partial D_{1} .\end{cases}
$$

Note that at Steps 2 and 4 of the flowchart, the volume potential $V_{\lambda, j}(x)$ and the iterate $w_{j+1}(x)$ are computed by quadratures of high accuracy with 864 Gauss points placed on the unit disk. The Gauss points are selected by using the Cartesian Gauss rule for the polar coordinate variables $(r, \theta)$ : dividing the unit disk into $4_{r} \times 24_{\theta}$ elements and using $3_{r} \times 3_{\theta}$ Gauss points in each element. The key step is the solution of the boundary integral equation for the simple-layer density $\eta_{j+1}$ in Step 3. In our numerical work, $\eta_{j+1}$ is solved by point collocation using piecewise constant boundary elements (i.e., $S_{h}^{1,0}(\partial \Omega)$-systems) with uniform mesh on $\partial \Omega$, where $\partial \Omega$ is divided into 64 equal pieces of arc (i.e., $h=2 \pi / 64$ ). Here collocation is taken as a measure of convenience, because the Galerkin boundary element method as stated in (4.10) and (4.12) involve significantly more programming work. It is also for this reason that in this section we have not attempted to confirm the error estimates given in $\S 5$ and $\S 6$ experimentally. We nevertheless hope that the examples furnished here will serve as useful benchmarks for researchers doing similar work, and that in the future we will also be able to derive and numerically verify error estimates for the boundary element point-collocation scheme.

In all our numerical computations, $\varepsilon=10^{-4}$ has been used as the relative $L_{2}$ error in solutions to terminate the iteration, see Flow-chart Step 5.

We first test the accuracy of our computer program this way. Choose a known (smooth) function $y(x), x \in \bar{\Omega}$, and for a somewhat arbitrary function $F$, where $F$ 
is nonlinear in $u$, we compute the solution of

$$
\begin{cases}\Delta u(x)-F(x, u(x))=0, & x \in \Omega, \\ u(x)=y(x), & x \in \partial \Omega\end{cases}
$$

where

$$
F(x, u(x))=F(u(x))+[\Delta y(x)-F(y(x))], \quad x \in \Omega .
$$

Obviously, $y(x)$ is an exact solution of the (artificially set example) above. We then use $y(x)$ as a benchmark to test whether we can numerically recover $u(x)=y(x)$. The computer results are affirmative: the output data have shown high accuracy.

In the following, we provide numerical examples which do not have exact solutions available. The accompanying theoretical discussions are to a large extent motivated by visualization of the numerical results.

Example 7.1. Multiple solutions - two stable solutions bounding an unstable one. Consider

$$
\begin{cases}\Delta u(x)-\gamma u(x)[u(x)-a][u(x)-1]=0, & x \in D_{1}, \\ u(x)=0, & x \in \partial D_{1},\end{cases}
$$

where $\gamma>0,0<a<1 / 2$. It is easy to see that $u(x)=\bar{v}(x) \equiv 0$ is a solution. One can further establish that $\bar{v}(x) \equiv 0$ is stable. This solution is of less interest because it is trivial.

Substituting $\bar{u}(x) \equiv 1$ for $u$ into (7.2), we also easily verify that $\bar{u}(x)$ is a supersolution. (Actually, $\bar{u}(x) \equiv C$ for any $C \geq 1$ will do.)

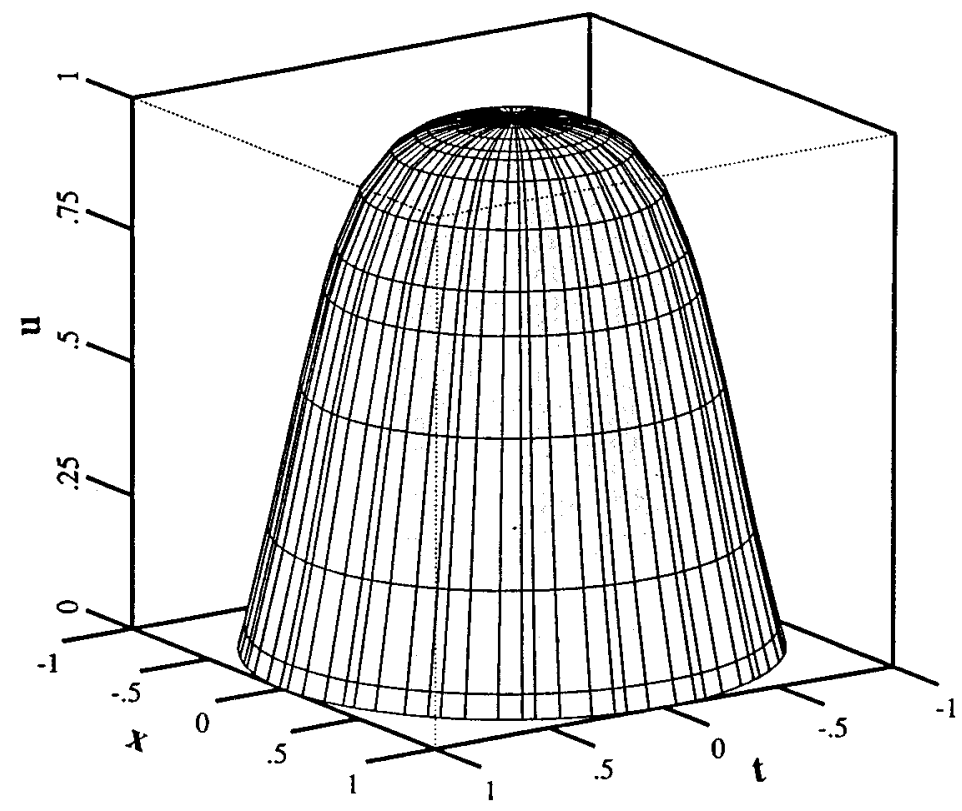

FiguRE 7.1. A bell-shaped stable positive boundary element numerical solution $u(x)$ satisfying $\Delta u-u\left(u-\frac{1}{4}\right)(u-1)=0$ and the zero Dirichlet boundary condition for Example 7.1. 
In $(7.2)$, we have

$$
\begin{aligned}
F(x, u) & \equiv F(u)=\gamma u(u-a)(u-1), \\
\frac{\partial F}{\partial u} & =\gamma[(u-a)(u-1)+u(u-1)+u(u-\alpha)] \\
& =\gamma\left[3 u^{2}-2(a+1) u+a\right] .
\end{aligned}
$$

In our computation, we choose

$$
\gamma=100, \quad a=\frac{1}{4}, \quad \text { yielding } \quad \max \frac{\partial F}{\partial u}=75, \quad u \in[0,1] .
$$

Thus, if we choose $\bar{u}(x) \equiv 1, \lambda=75.69$, then (3.10) is satisfied.

The monotone scheme beginning from $u_{0}(x)=\bar{u}(x) \equiv 1$ takes 30 iterations, yielding a bell-shaped function $u_{\infty}(x)$ as shown in Fig. 7.1. According to Theorem 2.2 , this is a stable solution. Since $u_{\infty}(x) \not \equiv 0 \equiv \bar{v}(x)=v_{\infty}(x)$, we know from Theorem 2.2 that there is at least one unstable solution of (7.2), called a "spike-layer" solution, sandwiched between $v_{\infty}$ and $u_{\infty}$.

Generally, it is known that for (7.2), the following are true:

(i) Any solution $u$ of (7.2) must satisfy $0 \leq u(x) \leq 1$;

(ii) For $\gamma>0$ sufficiently small, (7.2) has the trivial solution as its only solution;

(iii) The spike-layer solution exists only when $\gamma>0$ is large.

Meanwhile, the antimonotone convergence of the simple-layer densities, guaranteed by Theorem 3.1, is reflected in Fig. 7.2.

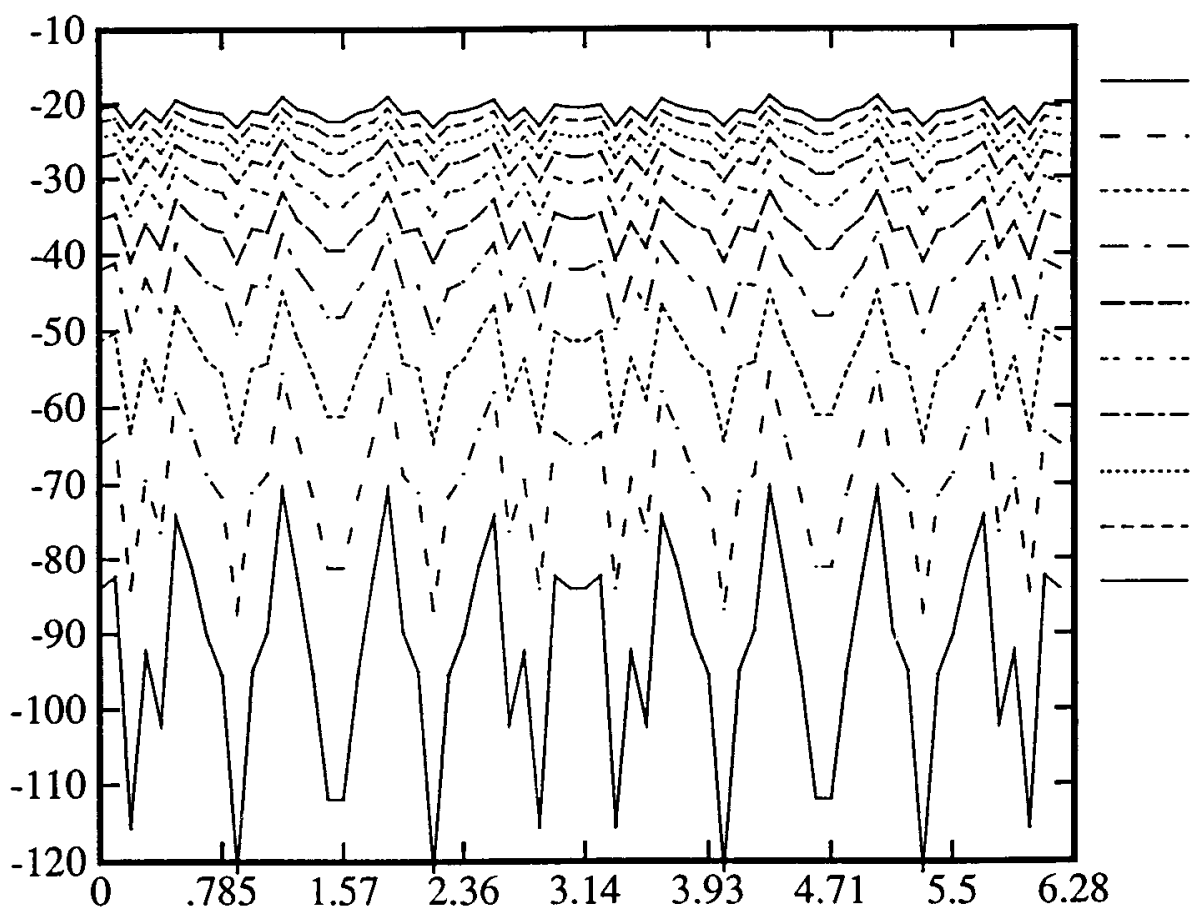

FiguRE 7.2. The antimonotone convergence of the simple-layer densities for Example 7.1. While the solutions $u_{h}^{n}(x)$ decrease monotonically on the unit disk, the simple-layer densities $\eta_{h}^{n}(x)$ increase monotonically for $n=1$ (bottom curve) to $n=10$ (top curve). 
Example 7.2. A monotone dissipative operator equation.

Consider

$$
\begin{cases}\Delta u(x)-4 u^{3}(x)=\gamma x_{1}^{2}, & x=\left(x_{1}, x_{2}\right) \in D_{1}, \quad \gamma>0 \\ u(x)=0, & x \in \partial D_{1}\end{cases}
$$

The nonlinear operator $\mathcal{N}$ on the left-hand side of the PDE in (7.1) is known to be a monotone dissipative operator as it satisfies

$$
\begin{aligned}
\left\langle\mathcal{N} u_{1}-\mathcal{N} u_{2}, u_{1}-u_{2}\right\rangle_{L^{2}\left(D_{1}\right)} & =\left\langle\left(\Delta u_{1}-4 u_{1}^{3}\right)-\left(\Delta u_{2}-4 u_{2}^{3}\right), u_{1}-u_{2}\right\rangle \\
& =\int_{D_{1}}\left[\Delta\left(u_{1}-u_{2}\right)-4\left(u_{1}^{3}-u_{2}^{3}\right)\right]\left(u_{1}-u_{2}\right) d x \\
& =-\int\left[\left|\nabla\left(u_{1}-u_{2}\right)\right|^{2}+4\left(u_{1}^{3}-u_{2}^{3}\right)\left(u_{1}-u_{2}\right)\right] d x \leq 0,
\end{aligned}
$$

for $u_{1}, u_{2} \in H_{0}^{2}\left(D_{1}\right)$. From the theory of monotone dissipative operators it is straightforward ([11], e.g.) to establish that (7.4) has a unique solution $u \in$ $C^{\infty}\left(\bar{D}_{1}\right)$.

From the way (7.4) is given, we can further prove that the solution satisfies $u(x)<0, x \in D_{1}$. Assume the contrary. Then by the usual maximum principle [13] $G \equiv\left\{x \in D_{1} \mid u(x)>0\right\}$ is nonempty. Thus, on $G, u$ satisfies

$$
\left\{\begin{array}{lc}
\Delta u(x)=4 u^{3}(x)+\gamma x_{1}^{2}>0, & x \in G, \\
u(x)>0, & x \in G, \\
u(x)=0, & x \in \partial G .
\end{array}\right.
$$

But for $u$ satisfying $\Delta u \geq 0$ on $G$ and $u=0$ on $\partial G$, the maximum principle implies that $u(x) \leq 0$ on $G$, a contradiction.

For (7.4), we have $F(x, u)=4 u^{3}+\gamma x_{1}^{2}$. For $D_{1}$, it is known that $\lambda_{1}$, the smallest eigenvalue of $-\Delta$ corresponding to the homogeneous Dirichlet condition, is (cf. [7, p. 188], e.g.)

$$
\lambda_{1} \approx(2.4048)^{2} \approx 5.783063
$$

Therefore,

$$
\frac{\partial F}{\partial u}=12 u^{2} \geq 0>-\lambda_{1} \approx-5.783063 .
$$

We may take $\bar{u}(x) \equiv 0$ as a supersolution, and $\bar{v}(x) \equiv-C, C>0$, as a subsolution, if $C$ is chosen such that $4 C^{3}-\gamma x_{1}^{2} \geq 0, \forall x \in \bar{D}_{1}$.

Because of (7.5), we easily see that $[\mathrm{H}]$ and (4.15) are satisfied for $\tilde{\gamma}_{1}<0$ if $\left|\tilde{\gamma}_{1}\right|$ is small.

In our numerical computations, we choose $\gamma=10$ and $\lambda=4$. The computation takes 8 iterations to converge. The numerical solution is plotted in Fig. 7.3. The reader can easily see that the solution $u(x)$ is nonpositive.

Note that Theorem 5.2 applies to Examples 7.1 and 7.2 . 


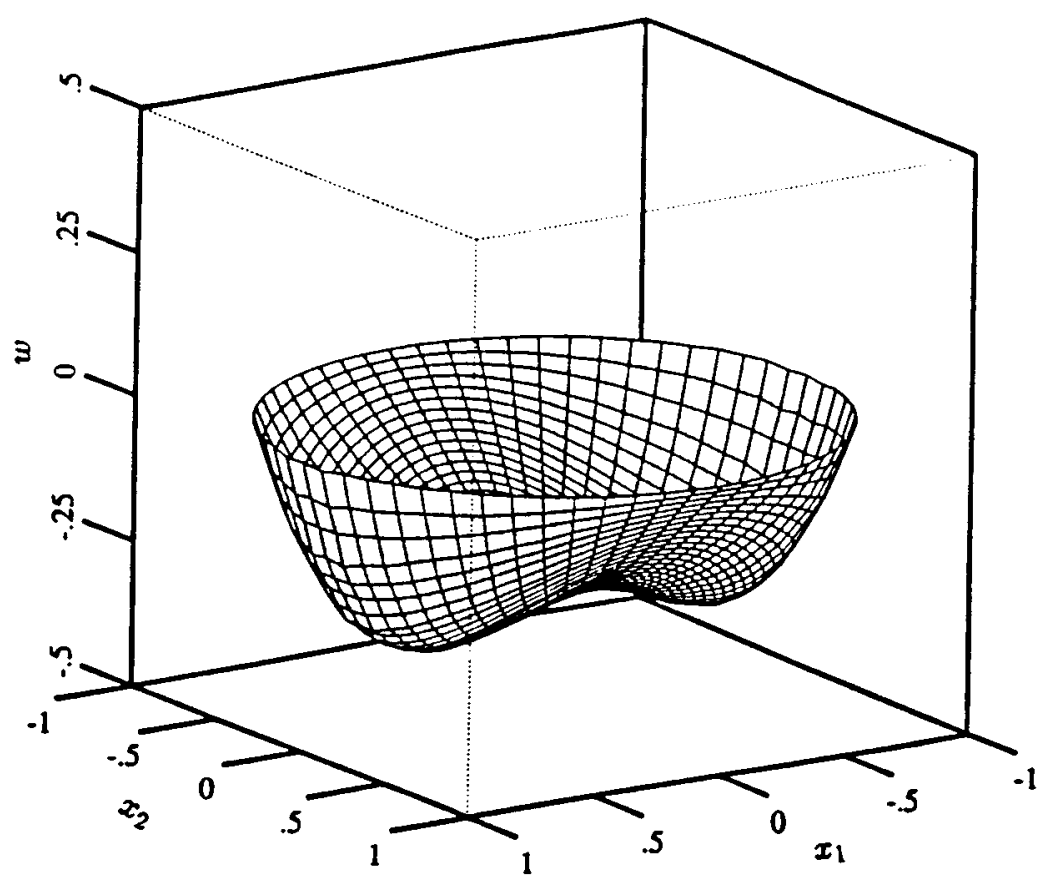

Figure 7.3. Boundary element numerical solution of $\Delta u-4 u^{3} \equiv 10 x_{1}^{2}$ subject to the zero Dirichlet boundary condition, Example 7.2.

Example 7.3. A nonlinear PDE of nonmonotone type whose existence or nonexistence of stable solutions of one sign depends on the size of forcing.

Consider

$$
\begin{cases}\Delta u(x)+4 u^{3}(x)=\gamma x_{1}^{2}, & x \in D_{1}, \quad \gamma>0 \\ u(x)=0, & x \in \partial D_{1} .\end{cases}
$$

Suppose we are interested in finding nonpositive solutions

$$
u(x) \leq 0 \quad \text { on } \quad D_{1} .
$$

This condition is now appended to (7.6). Later on, we will be able to remove (7.7) provided that $\gamma>0$ is sufficiently small, and that proper supersolutions and subsolutions are chosen; see (iii) below.

We now show the following.

(i) The system (7.6) and (7.7) has no solution if $\gamma$ is large.

Let $\phi_{1}$ be the first eigenfunction of $-\Delta$ satisfying

$$
\begin{cases}\Delta \phi_{1}+\lambda_{1} \phi_{1}=0 & \text { on } D_{1} \quad\left(\lambda_{1} \approx 5.783063\right), \\ \phi_{1}(x)>0 & \text { on } D_{1}, \\ \left.\phi_{1}\right|_{\partial D_{1}}=0 . & \end{cases}
$$

Here, $\phi_{1}(x)>0$ because we know that $\phi_{1}$ is of one sign on $D_{1}$ (cf. [6, §3.1], e.g.). 
From (7.6)-(7.8), we get

$$
\begin{aligned}
\gamma \int_{D_{1}} x_{1}^{2} \phi_{1}(x) d x= & \int_{D_{1}}\left[\Delta u(x)+4 u^{3}(x)\right] \phi_{1}(x) d x \\
= & \int_{D_{1}}\left[-\lambda_{1} u(x) \phi_{1}(x)+4 u^{3}(x)\right] \phi_{1}(x) d x \\
= & \int_{D_{1}} u(x) \phi_{1}(x)\left[4 u^{2}(x)-\lambda_{1}\right] d x \\
= & \int_{D_{1,1}} u(x) \phi_{1}(x)\left[4 u^{2}(x)-\lambda_{1}\right] d x \\
& +\int_{D_{1,2}} u(x) \phi_{1}(x)\left[4 u^{2}(x)-\lambda_{1}\right] d x
\end{aligned}
$$

where

$$
D_{1,1}=\left\{x \in D_{1} \mid-\sqrt{\lambda_{1}} / 2 \leq u(x) \leq 0\right\}, \quad D_{1,2}=\left\{x \in D_{1} \mid u(x)<-\sqrt{\lambda_{1}} / 2\right\} .
$$

Hence,

$$
0<\gamma \int_{D_{1}} x_{1}^{2} \phi_{1}(x) d x \leq \int_{D_{1,1}} u(x) \phi_{1}(x)\left[4 u^{2}(x)-\lambda_{1}\right] d x
$$

On $D_{1,1}$, we have $\sqrt{\lambda_{1}} / 2 \geq-u(x)$, and $\lambda_{1} \geq \lambda_{1}-4 u^{2}(x)$. Using the above in (7.9), we obtain

$$
\begin{gathered}
\frac{1}{2} \int_{D_{1,1}} \sqrt{\lambda_{1}} \phi_{1}(x) \lambda_{1} d x \geq \gamma \int_{D_{1}} x_{1}^{2} \phi_{1}(x) d x \\
\gamma \leq \frac{\lambda_{1}^{3 / 2} \int_{D_{1,1}} \phi_{1}(x) d x}{2 \int_{D_{1}} x_{1}^{2} \phi_{1}(x) d x} \leq \frac{\lambda_{1}^{3 / 2} \int_{D_{1}} \phi_{1}(x) d x}{2 \int_{D_{1}} x_{1}^{2} \phi_{1}(x) d x} .
\end{gathered}
$$

Thus, if $\gamma>0$ is chosen large enough to violate (7.10), the system (7.6) and (7.7) will not have a solution.

(ii) If the system (7.6) and (7.7) has a solution for $\gamma=\gamma_{1}>0$, then for all $\gamma$ : $0<\gamma<\gamma_{1}$, the system (7.6) and (7.7) also has a solution.

Let $\tilde{u}$ be the solution corresponding to $\gamma_{1}$ :

$$
\begin{cases}\Delta \tilde{u}(x)+4 \tilde{u}^{3}(x)=\gamma_{1} x_{1}^{2}, & x \in D_{1}, \\ \tilde{u}(x) \leq 0 & \text { on } D_{1}, \\ \tilde{u}(x)=0 & \text { on } \partial D_{1} .\end{cases}
$$

Consider $u$, satisfying

$$
\begin{cases}\Delta u(x)+4 u^{3}(x)=\gamma x_{1}^{2}, & x \in D_{1}, \quad 0<\gamma<\gamma_{1}, \\ u(x) \leq 0 & \text { on } D_{1}, \\ u(x)=0 & \text { on } \partial D_{1} .\end{cases}
$$


Thus, it is easy to check that $\bar{u}(x) \equiv 0$ is a supersolution for (7.12). We also claim that $\bar{v}(x)=\tilde{u}(x)$ is a subsolution, since

$$
\begin{aligned}
\Delta \bar{v}(x)+4 \bar{v}^{3}(x)-\gamma x_{1}^{2} & =\left[\Delta \tilde{u}(x)+4 \tilde{u}^{3}(x)-\gamma_{1} x_{1}^{2}\right]+\left(\gamma_{1}-\gamma\right) x_{1}^{2} \\
& =\left(\gamma_{1}-\gamma\right) x_{1}^{2} \geq 0 \quad \text { on } D_{1},
\end{aligned}
$$

and $\bar{v}(x)=\tilde{u}(x)=0$ on $\partial D_{1}$. By Theorem 2.3, a solution $u(x)$ of (7.12) exists, satisfying $\tilde{u}(x) \leq u(x) \leq 0$ on $D_{1}$.

(iii) The system (7.6) and (7.7) possesses a stable solution $\tilde{u}_{1}: \tilde{u}_{1}(x) \leq 0$ as well as a second solution $\tilde{u_{2}}: \tilde{u_{2}}(x)<\tilde{u_{1}}(x), x \in \Omega$, if $\gamma>0$ is sufficiently small.

We already know that $\bar{u}(x) \equiv 0$ is a supersolution of (7.6) and (7.7). We now construct a subsolution. Let $w(x)$ satisfy the Lane-Emden equation

$$
\begin{cases}\Delta w(x)+4 w^{3}(x)=0, & \text { on } D_{1}, \\ w(x)>0, & \text { on } D_{1}, \\ w(x)=0, & \text { on } \partial D_{1} .\end{cases}
$$

It is known (cf. [12], e.g.) that (7.14) has a unique solution (that is, also radially symmetric and monotonically decreasing in $|x|)$. Thus, we write $w(x)=w(r)$, $r=|x|, x \in D_{1}$. Define

$$
w_{\alpha}(x)=\alpha w(\alpha r), \quad 0<\alpha<1
$$

Then it is routine to verify that $w_{\alpha}(x)$ satisfies

$$
\Delta w_{\alpha}(x)+4 w_{\alpha}^{3}(x)=0, \quad w_{\alpha}(x) \geq \varepsilon>0, \text { for } \varepsilon=\alpha w(\alpha) \text { and } x \in D_{1} .
$$

Let

$$
\bar{v}(x)=-\beta w_{\alpha}(x), \quad 1>\beta>0, \text { for some fixed } \beta .
$$

Then

$$
\begin{aligned}
\Delta \bar{v}(x)+4 \bar{v}^{3}(x)-\gamma x_{1}^{2} & =-\beta \Delta w_{\alpha}(x)-4 \beta^{3} w_{\alpha}^{3}(x)-\gamma x_{1}^{2} \\
& =4 \beta w_{\alpha}^{3}(x)-4 \beta^{3} w_{\alpha}^{3}(x)-\gamma x_{1}^{2} \\
& =4 \beta\left(1-\beta^{2}\right) w_{\alpha}^{3}(x)-\gamma x_{1}^{2} \\
& \geq 4 \beta\left(1-\beta^{2}\right) \varepsilon^{3}-\gamma x_{1}^{2} \geq 0,
\end{aligned}
$$

if $\gamma>0$ is sufficiently small. Also,

$$
\bar{v}(x)=-\beta w_{\alpha}(x)=-\beta \alpha w(\alpha) \leq-\beta \alpha \varepsilon, \quad \text { if } \quad x \in \partial D_{1} .
$$

Thus $\bar{v}(x)$ is a subsolution, if $\gamma>0$ is sufficiently small. Hence the system (7.6) and (7.7) admit a stable solution for $\gamma>0$ sufficiently small. Call this solution $\tilde{u}_{1}$. From (ii), we further see that $\tilde{u}_{1} \uparrow 0$ uniformly as $\gamma \downarrow 0$.

To establish the existence of the second solution $\tilde{u_{2}}<\tilde{u_{1}}$ (on $\Omega$ ), we use a variational approach. For ease of discussion, set $z=-u$ in (7.6) and (7.7), giving

$$
\begin{cases}\Delta z(x)+4 z^{3}(x)+\gamma x_{1}^{2}=0, & \text { on } D_{1}, \\ z(x)>0, & \text { on } D_{1}, \\ z(x)=0, & \text { on } \partial D_{1} .\end{cases}
$$


Note that $z_{1}=-\tilde{u_{1}}$ is a solution of (7.16). Setting $w=z-z_{1}$ in (7.16), we obtain

$$
\left\{\begin{array}{l}
\Delta w(x)+\left[12 z_{1}^{2}(x)\right] w(x)+\left[12 z_{1}(x)\right] w^{2}(x)+4 w^{3}(x)=0, \quad x \in D_{1}, \\
w(x)=0, \quad x \in \partial D_{1}
\end{array}\right.
$$

We now look for a solution of (7.17) that further satisfies

$$
w(x)>0, \quad x \in D_{1} .
$$

When $\gamma>0$ is sufficiently small, $\left\|z_{1}\right\|_{L^{\infty}(\Omega)}$ is small. Thus $\left\|12 z_{1}^{2}\right\|_{L^{\infty}(\Omega)}<\lambda_{1}$ (cf. (7.8) for $\lambda_{1}$ ) is satisfied for small $\gamma>0$. Then an application of the usual Mountain-Pass Lemma [3, 12] yields a classical solution $w$ of (7.17) and (7.18). Hence, $\tilde{u}_{2} \equiv-\left(z_{1}+w\right)<\tilde{u}_{1}<0$ on $\Omega$ is a second solution.

For (7.6), we have

$$
F(x, u)=-4 u^{3}+\gamma x_{1}^{2}, \quad \frac{\partial F}{\partial u}=-12 u^{2} .
$$

Although the subsolution as given in (7.14) and (7.15) is not known explicitly, it can be verified that the conditions $[\mathrm{H}]$ and (4.15) are satisfied if $\lambda \geq 0$.

In our numerical experiments, we have chosen $\gamma=10$ and $\lambda=4$. The numerical iterates converge after 16 iterations. The graph of the numerical solution $\tilde{u}_{1}$ can be found in Fig. 7.4.

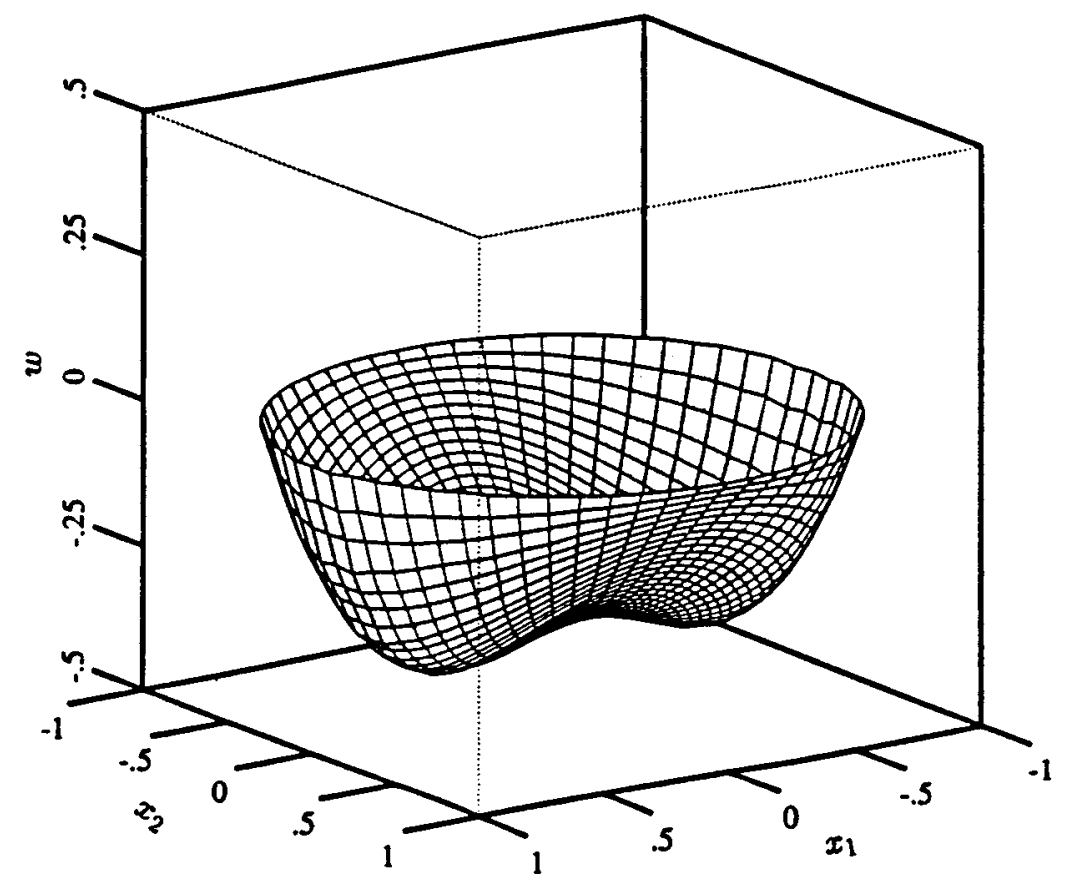

FigURE 7.4. Boundary element numerical solution of $\Delta u+4 u^{3}=10 x_{1}^{2}$ subject to the zero Dirichlet boundary condition, Example 7.3. 
Example 7.4. A nonlinear PDE of nonmonotone type with a unique stable nonpositive solution.

Consider

$$
\begin{cases}\Delta u(x)+4 u^{2}(x)=\gamma x_{1}^{2}, & x \in D_{1}, \quad \gamma>0 \\ u(x)=0, & x \in \partial D_{1} .\end{cases}
$$

It is easy to see that $\bar{u}(x) \equiv 0$ is a supersolution, and $\bar{v}(x) \equiv-C, C>0$, is a subsolution if $C$ is chosen such that $4 C^{2}-\gamma x_{1}^{2} \geq 0, \forall x_{1}:\left|x_{1}\right| \leq 1$. Thus, by Theorem 2.2, there exists a stable solution $u(x)$ such that $-C \leq u(x) \leq 0, \forall x \in D_{1}$. We now show that nonpositive solutions of (7.19) are unique. Suppose $u^{(1)}$ and $u^{(2)}$ both are nonpositive and satisfy (7.19). We may assume that

$$
u^{(1)}(x) \geq u^{(2)}(x) \quad \forall x \in D_{1}, \quad u^{(1)} \not \equiv u^{(2)} .
$$

Otherwise, by Theorem 2.2 and Remark 2.4(a), $\bar{v}(x)=\min \left\{u^{(1)}(x), u^{(2)}(x)\right\}, x \in$ $D_{1}$, is a subsolution of (7.19) and monotone iteration with $\bar{v}(x)$ as the initial state will yield a limit solution $u_{\infty}(x)$ such that $u^{(1)}(x) \geq u_{\infty}(x), \forall x \in D_{1}, u^{(1)} \not \equiv u_{\infty}$. From (7.19) and (7.20), we have

$$
\begin{aligned}
0 & =\int_{\partial D_{1}}\left[u^{(2)} \frac{\partial u^{(1)}}{\partial n}-u^{(1)} \frac{\partial u^{(2)}}{\partial n}\right] d \sigma=\int_{D_{1}}\left[u^{(2)} \Delta u^{(1)}-u^{(1)} \Delta u^{(2)}\right] d x \\
& =-\int_{D_{1}}\left[u^{(2)}\left(4 u^{(1)^{2}}-\gamma x_{1}^{2}\right)-u^{(1)}\left(4 u^{(2)^{2}}-\gamma x_{1}^{2}\right)\right] d x \\
& =-\int_{D_{1}}\left[4 u^{(1)} u^{(2)}\left(u^{(1)}-u^{(2)}\right)+\gamma x_{1}^{2}\left(u^{(1)}-u^{(2)}\right)\right] d x<0,
\end{aligned}
$$

a contradiction.

Thus, in contrast to Example 7.3, the size of $\gamma$ here does not affect the existence or nonexistence of the unique stable nonpositive solution. (Nevertheless, we do not rule out that some other stable solutions not of one sign may still exist.)

For (7.19), we have

$$
F(x, u)=-4 u^{2}+\gamma x_{1}^{2}, \quad \frac{\partial F}{\partial u}=-8 u .
$$

Note that we need only consider $u \leq 0$. Here, to ensure that $[\mathrm{H}]$ and (4.15) are satisfied, we need only use $\lambda=0$.

In our numerical computation, we choose $\gamma=10$ in (7.19) and use $\lambda=4$. The numerical solutions converge after 9 iterations. The limiting solution is plotted in Fig. 7.5.

Although the PDEs in Examples 7.2, 7.3 and 7.4 seem to have vastly different nonlinearities in a qualitative sense, we nevertheless notice that the solution graphics in Figs. 7.3, 7.4 and 7.5 seem to display similar profiles.

Example 7.5. A nonlinear PDE of nonmonotone type, whose stable solutions, not of one sign, may be obtained through symmetry.

Consider

$$
\begin{cases}\Delta u(x)+4 u^{3}(x)=\gamma x_{1}^{3}, & x \in D_{1}, \quad \gamma>0 \\ u(x)=0, & x \in \partial D_{1} .\end{cases}
$$




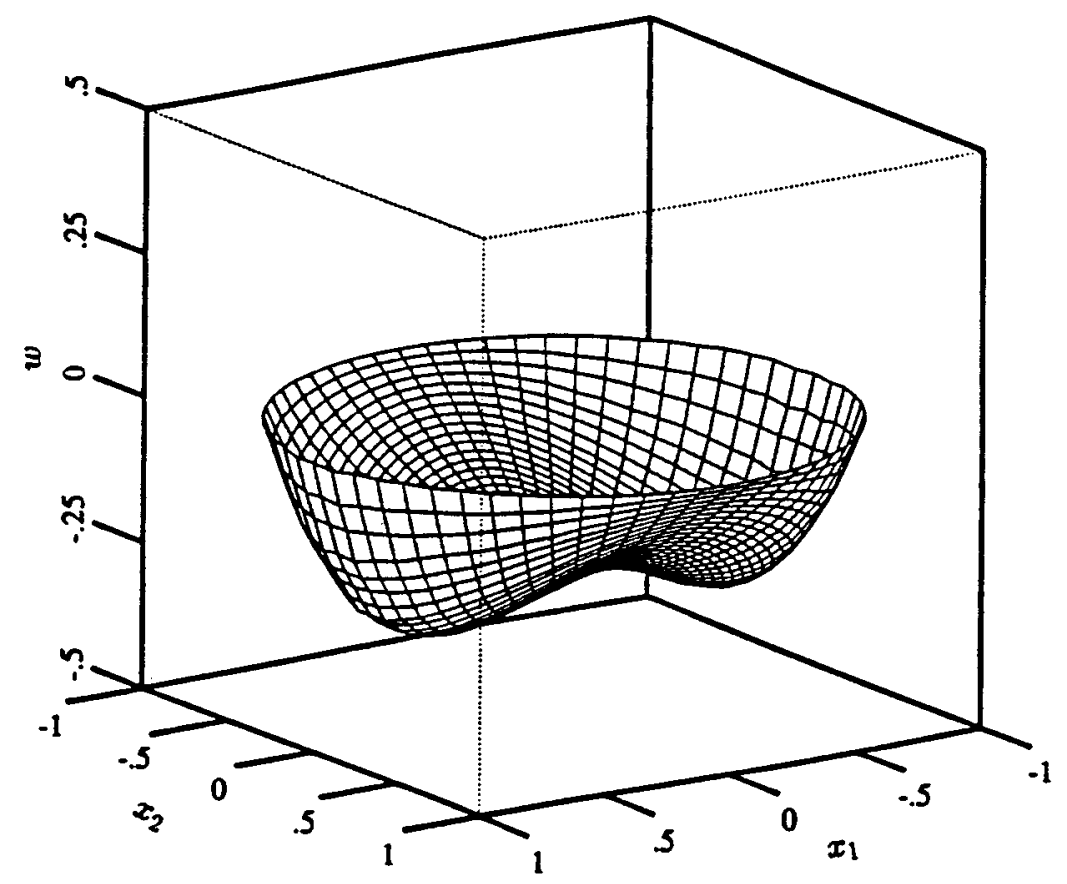

FiguRe 7.5. Boundary element numerical solution of $\Delta u+4 u^{2}=10 x_{1}^{2}$ subject to the zero Dirichlet boundary condition, Example 7.4.

Here the forcing term $\gamma x_{1}^{3}$ changes sign as $x$ moves from $D_{1,-}$ into $D_{1,+}$ :

$$
D_{1,-} \equiv\left\{x \in D_{1} \mid x_{1}<0\right\}, \quad D_{1,+} \equiv\left\{x \in D_{1} \mid x_{1}>0\right\} .
$$

Note that, if $u(x)$ is a solution of $(7.22)$, then so is $-u\left(-x_{1}, x_{2}\right)$, i.e., $u$ is antisymmetric with respect to the $x_{2}$-axis, and thus we deduce that $u(x)=0$, $x \in \bar{D}_{1,-} \cap \bar{D}_{1,+}$. So we need only consider the boundary value problem

$$
\begin{cases}\Delta \tilde{u}(x)+4 \tilde{u}^{3}(x)=\gamma x_{1}^{3}, & x \in D_{1,+} \\ \tilde{u}(x)=0, & x \in \partial D_{1,+}\end{cases}
$$

because on $D_{1,-}$ we simply have $u\left(x_{1}, u_{2}\right)=-\tilde{u}\left(-x_{1}, x_{2}\right)$ for $x \in D_{1,-}$. The nonlinear boundary value problem (7.23) is similar to the one considered in (7.6) because the forcing terms $\gamma x_{1}^{3}$ and $\gamma x_{1}^{2}$ are all nonnegative on each domain. So essentially all the arguments in parts (i)-(iii) of Example 7.3 apply. The only concern one might have for (7.23) may be that $\partial D_{1,+}$ is not a $C^{\infty}$ curve. Nevertheless, $\partial D_{1,+}$ is Lipschitz continuous. With suitable modifications, the arguments still go through and there will be no problems.

In our numerical computations, we again choose $\gamma=10$ in (7.22) and use $\lambda=4$. The numerical solutions converge after 13 iterations, using $u_{0}(x) \equiv 0$. The graphics is given in Fig. 7.6. The reader will find that the solution changes sign across the $x_{2}$-axis. 


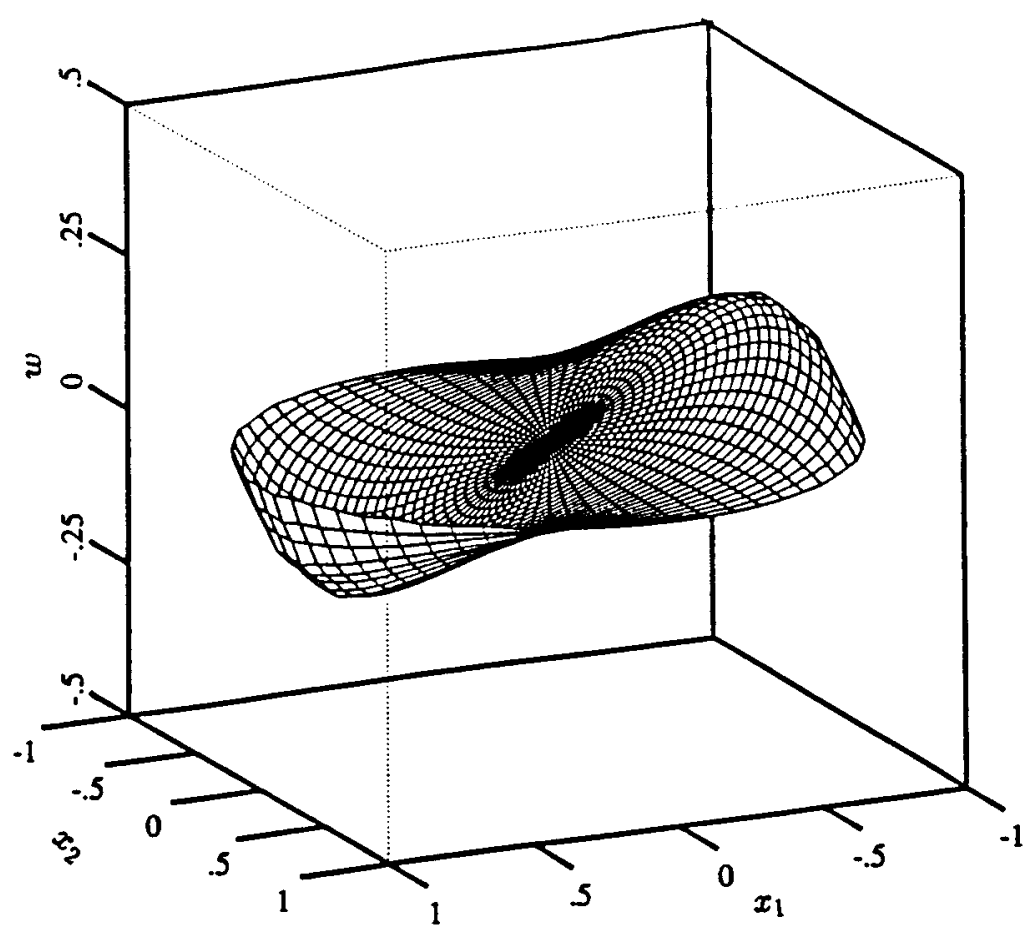

FiguRE 7.6. Boundary element numerical solution of $\Delta u+4 u^{3}=10 x_{1}^{3}$ subject to the zero Dirichlet boundary condition, Example 7.5.

Remark 7.6. How restrictive is the working assumption $[\mathrm{H}]$ made in $\S 4$ ? From Examples 7.1-7.5, we see that for the monotone equation (7.4) in Example 7.2, $[\mathrm{H}]$ is always satisfied. The BVP (7.4) also has the least trouble with existence and uniqueness. For the BVP in Example 7.1, when $\gamma$ is sufficiently small, condition $[\mathrm{H}]$ holds. However, $[\mathrm{H}]$ fails when $\gamma$ is large, because the system (7.1) has multiple solutions that can be separated only by nonconstant sup- and subsolutions. So it appears that an alternative assumption to $[\mathrm{H}]$ is required. Nevertheless, computationally we are still able to obtain convergent numerical solutions.

For Examples 7.3 and 7.4, even though the existence/nonexistence and uniqueness/multiplicity of solutions are harder to determine, condition $[\mathrm{H}]$ can still largely be confirmed. In any case, regardless of whether $[\mathrm{H}]$ can be confirmed a priori or a posteriori, numerical solutions can always be generated by the computer and motivate us to establish further theoretical properties of solutions as given in this section. This is an indication of the usefulness of the development of a combination of theory (i.e., monotone iterations) and numerical methods (i.e., BEM).

\section{REFERENCES}

1. M. Abramowitz and I.A. Stegun, Handbook of Mathematical Functions, Dover, New York, 1965. MR 34:8607

2. H. Amann, Supersolution, monotone iteration and stability, J. Diff. Eq. 21 (1976), 367-377. MR 53:11226

3. A. Ambrosetti and P. Rabinowitz, Dual variational methods in critical point theory and applications, J. Funct. Anal. 14 (1973), 349-381. MR 51:6412 
4. I. Babuška and A.K. Aziz, The Mathematical Foundations of the Finite Element Method with Applications to Partial Differential Equations, Academic Press, New York, 1972. MR 54:9111

5. C.A. Brebbia and S. Walker, Boundary Element Techniques in Engineering, NewnesButterworths, London, 1980.

6. G. Chen and J. Zhou, Boundary Element Methods, Academic Press, London, 1992. MR 93e: 65143

7. G. Chen and J. Zhou, Vibration and Damping in Distributed Systems, Vol. II: WKB and Wave Methods, Visualization and Experimentation CRC Press, Boca Raton, Florida, 1993.

8. Y. Deng, Boundary element methods for nonlinear partial differential equations, Ph.D. dissertation, Math. Dept., Texas A\&M Univ., College Station, Texas, August 1994.

9. G. Fichera, Proc. of Symposium on Partial Differential Equations and Continuum Mechanics ( R.E. Langer, ed.), 55-80, Univ. of Wisconsin Press, Madison, Wisconsin, 1961. MR 27:6016

10. G.C. Hsiao and W.L. Wendland, The Aubin-Nitsche lemma for integral equations, J. Integral Eq. 3 (1981), 299-315. MR 83j:45019

11. J.L. Lions, Quelques Méthodes de Résolution des Problèmes aux Limites Non Lineaires, Dunod, Paris, 1969. MR 41:4326

12. W.M. Ni, Lecture Notes published by Institute of Mathematics, National Tsing Hua Univ., Hsinchu, Taiwan, Rep. of China, May, 1987.

13. M. Protter and H. Weinberger, Maximum Principles in Differential Equations, Prentice-Hall, Englewood Cliffs, New Jersey, 1967. MR 36:2935

14. K. Ruotsalainen and J. Saranen, On the convergence of the Galerkin method for nonsmooth solutions of integral equations, Num. Math. 54 (1988), 295-302. MR 90a:65281

15. K. Ruotsalainen and W.L. Wendland, On the boundary element method for a nonlinear boundary value problem, Boundary Elements IX (C.A. Brebbia, ed.), Springer-Verlag, New York, 1987, pp. 385-393. MR 90e:65161

16. M. Sakakihara, An iterative boundary integral equation method for mildly nonlinear elliptic partial differential equations, Boundary Elements VII (C.A. Brebbia and G. Maier, ed.), vol. . II, Springer-Verlag, Berlin-Heidelberg, 1985, pp. 13.49-13.58. CMP 20:14

17. D. Sattinger, Topics in Stability and Bifurcation Theory, Lecture Notes in Mathematics, vol. . 309, Springer-Verlag, New York, 1973. MR 57:3569

(Y. Deng, G. Chen, and J. Zhou) Department of Mathematics, Texas A\&M University, College Station, Texas 77843

E-mail address: ydeng@cs.tamu.edu

E-mail address: gchen@math.tamu.edu

E-mail address: jzhou@math.tamu.edu

(W.-M. Ni) School of Mathematics, University of Minnesota, Minneapolis, Minnesota 55455 